\title{
Simulation-based Bayesian inference for latent traits of item response models: Introduction to the ltbayes package for $R$
}

\author{
Timothy R. Johnson • Kristine M. Kuhn
}

Published online: 27 November 2014

(C) Psychonomic Society, Inc. 2014

\begin{abstract}
This paper introduces the ltbayes package for R. This package includes a suite of functions for investigating the posterior distribution of latent traits of item response models. These include functions for simulating realizations from the posterior distribution, profiling the posterior density or likelihood function, calculation of posterior modes or means, Fisher information functions and observed information, and profile likelihood confidence intervals. Inferences can be based on individual response patterns or sets of response patterns such as sum scores. Functions are included for several common binary and polytomous item response models, but the package can also be used with user-specified models. This paper introduces some background and motivation for the package, and includes several detailed examples of its use.
\end{abstract}

Keywords Item response theory $\cdot$ Bayesian statistics $\cdot R$

\section{Introduction}

Item response models are a broad family of statistical models for item response distributions as a function of item- and respondent-specific parameters (Birnbaum 1968;

T. R. Johnson $(\varangle)$

Department of Statistical Science, University of Idaho, 875 Perimeter Drive MS 1104, Moscow, ID 83844-1104, USA

e-mail: trjohns@uidaho.edu

\section{K. M. Kuhn}

Department of Management, Information Systems, and Entrepreneurship, Washington State University, Pullman, WA 99164-4736, USA

e-mail:kmkuhn@wsu.edu
Lord 1952, 1953). One key property of these models is the separation of the model parameters into invariant itemand respondent-specific parameters so that items can be scaled independently of respondents, and similarly respondents can be scaled independently of items. Whereas each item may be characterized by several parameters, respondents are usually characterized by a single parameter or latent trait, except in multidimensional models where two or more latent traits are used. Similarly, inference for item response models frequently treats the problems of item parameter and latent trait estimation separately. This is due in part to certain technical aspects of the inferential methods that are used, but also because of differing objectives in terms of whether the interest is in the characteristics of the items or the respondents. The focus here is on the latter problem-i.e., inferences about the latent traits that scale the respondents.

The objective of this paper is to serve as an introduction to the Itbayes package (Johnson 2014) for R (R Core Team 2013). This package was specifically designed to meet two goals: to provide a flexible set of functions for investigating latent trait posterior distributions or likelihoods for a variety of item response models including user-specified models, and to provide a means to base such inferences not only on response patterns but also on sets of response patterns such as sum scores. This latter goal is motivated by some theoretical results concerning stochastic ordering that have some useful practical implications for the use of sum scores. Together, these can be very useful not just for researchers and methodologists but also for communicating inferences concerning respondents to practitioners. This introduction provides a brief review of item response models, latent trait estimation, and stochastic ordering by sum scores. The rest of the paper describes and illustrates the functionality of the ltbayes package. 
Item response models

Here we will focus on discrete item responses where $Y_{i j}=$ $0,1, \ldots, r-1$ where $r \geq 2$ denotes the number of item response categories which includes binary $(r=2)$ and polytomous $(r>2)$ item response models. Responses are assumed to vary over respondents $(i=1,2, \ldots, n)$ and items $(j=1,2, \ldots, m)$. Item response models characterize the joint distribution of the $m$ item responses $Y_{i 1}, Y_{i 2}, \ldots, Y_{i m}$ for each respondent. However, because the item responses are assumed to be conditionally independent given a latent trait $\zeta_{i}$, the model reduces to one for the conditional distribution of each $Y_{i j}$ of the form $P\left(Y_{i j}=y \mid \zeta_{i}, \boldsymbol{\eta}_{j}\right)$, where $\boldsymbol{\eta}_{j}$ is a vector of one or more item-specific parameters. For example, the two-parameter binary logistic model (Birnbaum 1968) is

$P\left(Y_{i j}=y \mid \zeta_{i}, \eta_{j}\right)=\frac{\exp \left[y \alpha_{j}\left(\zeta_{i}-\beta_{j}\right)\right]}{1+\exp \left[\alpha_{j}\left(\zeta_{i}-\beta_{j}\right)\right]}$,

for $y=0,1$ where the elements of $\boldsymbol{\eta}_{j}$ are $\alpha_{j}$ and $\beta_{j}$, the item discrimination and difficulty parameters, respectively. For a polytomous example, the partial credit model (Masters 1982) is

$$
P\left(Y_{i j}=y \mid \zeta_{i}, \boldsymbol{\eta}_{j}\right)=\frac{\exp \left(y \zeta_{i}-\sum_{k=0}^{y} \beta_{j k}\right)}{\sum_{k=0}^{r-1} \exp \left(k \zeta_{i}-\sum_{l=0}^{k} \beta_{j l}\right)},
$$

for $y=0,1,2, \ldots, r-1$ where $\beta_{j 0}=0$. Here $\beta_{j k}$ is the item difficulty parameter for item $j$ when conditioning on a response in the $k$-th set of adjacent response categories. These and other item response models are reviewed in the Appendix.

Inferences for item response models can often be viewed as being based in some way on the probability model

$h(\boldsymbol{\omega}, \boldsymbol{\eta}) \prod_{i=1}^{n} g\left(\zeta_{i} \mid \boldsymbol{\omega}\right) \prod_{j=1}^{m} P\left(Y_{i j}=y_{i j} \mid \zeta_{i}, \boldsymbol{\eta}_{j}\right)$,

where $g\left(\zeta_{i} \mid \omega\right)$ is the distribution of $\zeta_{i}$ with parameter(s) $\boldsymbol{\omega}, \boldsymbol{\eta}$ is a vector of the distinct elements of $\boldsymbol{\eta}_{1}, \boldsymbol{\eta}_{2}, \ldots, \boldsymbol{\eta}_{m}$, and $h(\boldsymbol{\omega}, \boldsymbol{\eta})$ is a prior distribution on $\boldsymbol{\omega}$ and $\boldsymbol{\eta}$. This is the joint distribution of the parameters and item responses, and is therefore proportional to the posterior distribution of the item parameters and latent traits given the item responses, which provide a fully Bayesian approach to the estimation of both sets of parameters. Alternatively, marginal maximum likelihood (Bock and Aitkin 1981; Bock and Lieberman 1970) or conditional maximum likelihood (Andersen 1970) can be used to obtain estimates of item parameters without estimating the latent traits. Marginal maximum likelihood implicitly specifies a flat prior distribution on any hyperparameters and item parameters (but not $\zeta_{i}$ ) such that $h(\omega, \eta) \propto 1$, and integrates the joint likelihood with respect to the distribution of $\zeta_{i}$ to eliminate the latent traits from the estimation problem as nuisance variables. Conditional maximum likelihood also implicitly specifies a flat prior and eliminates the latent traits from the likelihood by conditioning on sufficient statistics (when they exist).

\section{Latent trait estimation}

Estimation of the latent trait $\zeta$ is typically based in some way on the posterior distribution

$k(\zeta \mid \boldsymbol{y}, \boldsymbol{\eta}, \boldsymbol{\omega}) \propto g(\zeta \mid \boldsymbol{\omega}) \prod_{j=1}^{m} P\left(Y_{j}=y_{j} \mid \zeta, \boldsymbol{\eta}_{j}\right)$,

where $\boldsymbol{y}=\left(y_{1}, y_{2}, \ldots, y_{m}\right)^{\prime}$ denotes the realizations of $m$ item responses - one to each item by a real or hypothetical respondent - often referred to as a response pattern. (Where there is no loss of clarity, we will drop the subscript $i$ for simplicity.) The right-hand side of Eq. 1 has two components: the latent trait prior distribution $g(\zeta \mid \omega)$, which may depend on one or more hyper-parameters $\omega$, and the likelihood function, which depends on the item parameters $\boldsymbol{\eta}_{j}$. This posterior implies that $\omega$ and $\boldsymbol{\eta}$ are known, or at least very precisely estimated so that a prior would be nearly degenerate with its mass concentrated at $\omega$ and $\eta$. This situation is relatively common in practice, however, because $n$ is usually several orders of magnitude larger than $m$ leading to relatively precise item parameter estimates and the creation of banks of items with (nearly) known parameters. Similarly, large-scale testing can provide relatively accurate estimates of $\omega$. In any case, here we focus only on the case where $\omega$ and $\eta$ are known or specified a priori.

There are three common point estimators of $\zeta$. Two Bayesian approaches are the posterior mode or the "maximum a posteriori” (MAP) estimate defined as

$\tilde{\zeta}=\underset{\zeta}{\arg \max } k(\zeta \mid \boldsymbol{y}, \boldsymbol{\eta}, \boldsymbol{\omega})$,

and the posterior mean or "expected a posteriori" (EAP) estimator

$\ddot{\zeta}=\int \zeta k(\zeta \mid \boldsymbol{y}, \boldsymbol{\eta}, \boldsymbol{\omega}) d \zeta$.

A third non-Bayesian estimator is the maximum likelihood estimator (MLE) based on the likelihood function in Eq. 1 and defined as

$\hat{\zeta}=\underset{\zeta}{\arg \max } \prod_{j=1}^{m} P\left(Y_{j}=y_{j} \mid \zeta, \boldsymbol{\eta}_{j}\right)$.

The MLE is equivalent to the posterior mode when specifying a flat prior for $\zeta$ such that $g(\zeta \mid \omega) \propto 1$.

When using the MLE to estimate $\zeta$, the Fisher information function is an important tool for evaluating the 
relative precision of items and tests. Assuming that the log-likelihood function is twice-differentiable, the Fisher information function for an item response model is defined as

$I(\zeta)=-E\left[\frac{\partial^{2}}{\partial \zeta^{2}} \sum_{j=1}^{m} \log P\left(Y_{j}=y_{j} \mid \zeta, \boldsymbol{\eta}_{j}\right)\right]$,

where the expectation is with respect to the joint distribution of $Y_{i 1}, Y_{i 2}, \ldots, Y_{i m}$ given $\zeta$ and $\boldsymbol{\eta}$. For binary and polytomous item response models where the item response has a binomial or multinomial distribution, respectively, this can be written in closed form as

$I(\zeta)=\sum_{j=1}^{m} \sum_{y=0}^{r-1} I_{j y}(\zeta) P_{j y}(\zeta)$,

where $P_{j y}(\zeta)=P\left(Y_{j}=y \mid \zeta, \eta_{j}\right)$ and

$I_{j y}(\zeta)=\frac{P_{j y}^{\prime}(\zeta)^{2}-P_{j y}(\zeta) P_{j y}^{\prime \prime}(\zeta)}{P_{j y}(\zeta)^{2}}$

where $P_{j y}^{\prime}(\zeta)=\partial P_{j y}(\zeta) / \partial \zeta$ and $P_{j y}^{\prime \prime}(\zeta)=\partial^{2} P_{j y} / \partial \zeta^{2}$ (Samejima 1969; Bock 1972; Baker and Kim 2004). Because Eq. 3 can be decomposed into item- and categoryspecific components, $I_{j y}(\zeta)$ and $\sum_{y=0}^{r-1} I_{j y}(\zeta) P(\zeta)$ are often referred to as the category and item information functions, respectively. For clarity, $I(\zeta)$ is then referred to as the test information function.

Point and interval estimates for $\zeta$ can be motivated by standard asymptotic results. For example, a confidence interval for $\zeta$ can be specified as

$\hat{\zeta} \pm \frac{z_{\alpha / 2}}{\sqrt{I(\hat{\zeta})}}$

where $z_{\alpha / 2}$ is the $(1-\alpha / 2) \times 100 \%$-th percentile from a standard normal distribution. Posterior distributions for $\zeta$ also exhibit asymptotic normality for both binary (Chang and Stout 1993) and polytomous (Chang 1996) models under fairly general conditions. Unfortunately, finite sample properties of point estimates of $\zeta$ are not necessarily favorable. Several researchers have documented analytical and empirical evidence for bias of the MLE, MAP, and EAP estimators (e.g., Lord 1983; Warm 1989). Also, coverage rates of confidence intervals such as Eq. 4 may also be suspect, even when corrected for bias. Asymptotic results are with respect to the number of items (i.e., as $m \rightarrow \infty$ ). For tests with many items such as educational tests, $m$ may be sufficiently large for satisfactory finite sample behavior. However, for shorter tests, such as attitude or personality inventories, or short educational tests, finite sample behavior may be suspect. An alternative approach that is exact, even for finite samples, is a fully Bayesian approach that uses the full posterior distribution of $\zeta$, not just the posterior mean or mode (Van der Linden and Pashley 2010). The focus of the Itbayes package is on using the full posterior distribution, although it includes the means to compute maximum likelihood estimates and confidence intervals using Fisher information.

\section{Sum scores}

In item response theory, the scale for respondents is that of the latent trait $\zeta$, but those without an understanding of psychometric theory may find the scale of the sum score, $\tilde{Y}=\sum_{j=1}^{m} Y_{j}$, more intuitive. Sijtsma and Hemker (2000, pp. 392) note that

"...since the interpretation of $\zeta$ is rather complex and remote from everyday experience, the $\zeta$ scale may not be convenient for communicating test performance results to measurement practitioners (such as test constructors and psychologists who administer tests) and their clients (such as organizations and government institutions and the individuals tested at their request) and to pupils and their teachers and parents ... a more fruitful approach to communicating test results may be the use of simpler scales, and then $[\tilde{Y}]$ is a likely candidate. This is not to say that $[\tilde{Y}]$ or another summary score based on observable item scores have a self-evident meaning, but those scores are more familiar due to their direct relation to "doing things right or wrong" (abilities, achievements) or "earning more or fewer points" (personality traits, attitudes) than $[\zeta]$ scores which lack such a direct relation."

The simplicity and intuitiveness of sum scores and a lack of background in psychometric theory are perhaps why sum scores are frequently used in lieu of latent traits by not just practitioners but also some researchers.

While sum scores may be more intuitive, a significant advantage of item response theory is the concept of the latent trait. Fortunately, there are several theoretical results that bridge the gap between these two scales. Several researchers have established results concerning the conditional distributions of $\zeta$ given $\tilde{Y}$, and $\tilde{Y}$ given $\zeta$, for a variety of binary (Grayson 1988; Huynh 1994) and polytomous (Hemker et al. 1996, 1997, 2001; Van der Ark 2005; Van der Ark and Bergsma 2010) item response models (see Sijtsma and Hemker 2000, and Van der Ark, 2001, for reviews). Of particular interest here is the property of stochastic ordering of the latent trait by the sum score, meaning that if $\tilde{y}_{1}<\tilde{y}_{2}$ then

$P\left(\zeta>z \mid \tilde{Y}=\tilde{y}_{1}\right) \leq P\left(\zeta>z \mid \tilde{Y}=\tilde{y}_{2}\right)$

for any $z$. Stochastic ordering is implied by the property that a model has a monotone likelihood ratio (MLR), meaning 
that if $\tilde{y}_{1}<\tilde{y}_{2}$ then

$u\left(z, \tilde{y}_{1}, \tilde{y}_{2}\right)=\frac{P\left(\tilde{Y}=\tilde{y}_{1} \mid \zeta=z\right)}{P\left(\tilde{Y}=\tilde{y}_{2} \mid \zeta=z\right)}$

is a non-decreasing function of $z$. MLR implies a stochastic ordering of $\zeta$ by $\tilde{Y}$, and also of $\tilde{Y}$ by $\zeta$. Hemker et al. 1997 refer to these as stochastic ordering of the latent trait (SOL) and stochastic ordering of the manifest variable (SOM), respectively. Since the sum score is observed but $\zeta$ is not, SOL is the useful property for making inferences concerning latent traits using sum scores. All binary item response models with monotonic item response functions exhibit MLR and thus SOL (Grayson 1988; Huynh 1994), but of the polytomous models only the partial credit (Masters 1982) and rating scale (Andersen 1977; Andrich 1978a, b) models have the properties of MLR or SOL (Hemker et al. 1996, 1997, 2001). Van der Ark (2005), however, showed that polytomous models that do not guarantee SOL may still exhibit it in practice. Also, Van der Ark and Bergsma (2010) showed that all ordinal polytomous item response models exhibit what they termed weak SOL defined as

$P(\zeta>z \mid \tilde{Y}<\tilde{y}) \leq P(\zeta>z \mid \tilde{Y} \geq \tilde{y})$

for all $z$ and $\tilde{y}=1,2, \ldots, m(r-1)$.

Stochastic ordering of the latent trait by the sum score has some useful practical implications for the use of sum scores to make inferences concerning latent traits. SOL implies that it is reasonable to rank respondents with respect to their sum scores, while weak SOL implies that respondents can be grouped by "dichotomizing" their sum scores at any point. Furthermore, the expectations of the latent traits can be ordered with respect to sum scores when $\zeta$ is regressed on $\tilde{Y}$. This is because SOL implies

$E\left(\zeta \mid \tilde{Y}=\tilde{y}_{2}\right) \geq E\left(\zeta \mid \tilde{Y}=\tilde{y}_{1}\right)$,

if $\tilde{y}_{2}>\tilde{y}_{1}$, while weak SOL implies

$E(\zeta \mid \tilde{Y} \geq \tilde{y}) \geq E(\zeta \mid \tilde{Y}<\tilde{y})$

for $\tilde{y}=1,2, \ldots, m(r-1)$. It is important to note, however, that while (weak) stochastic ordering shows that it is consistent with the concept of the latent trait to scale respondents with respect to their sum scores, it is not statistically optimal in the sense that there can be some loss of information except in models where the sum score is a sufficient statistic for the latent trait (i.e., Rasch models). The optimal approach is to condition on response patterns. We suggest, however, that posterior distributions of latent traits given sum scores are useful to help communicate to laypersons the connection between the more intuitive sum score and the less intuitive latent trait, promoting a better understanding and appreciation of the latter. Doing so does not preclude the more statistically optimal methods. We agree with Sijtsma and Hemker (2000) that "nothing prevents the simultaneous use of IRT for test construction and the information function for measurement evaluation of $[\zeta]$ on the one hand, and the communication of test performance to measurement practitioners and laymen by means of scores such as $[\tilde{Y}]$, on the other hand" (pp. 383). Another issue to consider is that some researchers and practitioners will use sum scores rather than response patterns in contravention to psychometric theory. For these cases, it is important to understand how much (or how little) information is lost by doing so.

In some cases, it may only be possible to consider the posterior distribution of the latent trait given a sum score in lieu of a response pattern that is not available. This can occur when the item responses are lost during data processing and dissemination, or when they are not released as a method of statistical disclosure control to protect respondent confidentiality (Johnson 2013). This results in a kind of missing data problem where the individual item responses are missing but their sum is observed. Fortunately, the sum scores still contain some information and, as implied by the results on stochastic ordering, can rationally (although not optimally) be used to rank respondents with respect to their latent trait.

\section{Conditioning on sum scores}

The posterior distribution of $\zeta$ given a sum score $\tilde{Y}=\tilde{y}$ can be obtained as an extension of Eq. 1. Let $S$ denote the set of response patterns for a given sum score. The posterior distribution of $\zeta$ given the sum score is then

$h(\zeta \mid \tilde{y}, \boldsymbol{\eta}, \boldsymbol{\omega}) \propto g(\zeta \mid \boldsymbol{\eta}) \sum_{z \in S} \prod_{j=1}^{m} P\left(Y_{j}=z_{j} \mid \zeta, \eta_{j}\right)$.

Note that the summation is over the elements of $S$, where the response pattern $z^{\prime}=\left(z_{1}, z_{2}, \ldots, z_{m}\right)$ is an element of $S$ if and only if $\tilde{y}=\sum_{j=1}^{m} z_{j}$. Different posterior distributions can be specified by the definition of $S$. The posterior distribution of $\zeta$ given $\tilde{y}$ is given by defining $S$ as

$S=\left\{z \mid \sum_{j=1}^{m} z_{j}=\tilde{y}\right\}$

so that $S$ is the set of all response patterns that yield the sum score $\tilde{y}$. For another example, the conditional distribution of $\zeta$ given that the sum score is at least $\tilde{y}$ is obtained if

$S=\left\{z \mid \sum_{j=1}^{m} z_{j} \geq \tilde{y}\right\}$

so that now $S$ is the set of all response patterns that yield a sum score of at least $\tilde{y}$. The set of response patterns can also be defined as a single response pattern $\boldsymbol{y}=$ $\left(y_{1}, y_{2}, \ldots, y_{m}\right)^{\prime}$ such that

$S=\left\{z \mid z_{j}=y_{j}, j=1,2, \ldots, m\right\}$. 
Thus, the posterior distribution defined in Eq. 1 can be viewed a special case of that in Eq. 5.

\section{The Itbayes package}

As discussed earlier, inferences for $\zeta$ can often be based on its posterior distribution as in Eqs. 1 or 5, but this distribution is not typically amenable to analytical analysis. Some properties of the posterior distribution such as its mode can be obtained numerically, but other properties are more easily obtained using simulation-based methods. Simulated realizations from the posterior distribution of $\zeta$ are generated as a by-product of Markov chain Monte Carlo (MCMC) algorithms for Bayesian inference for item response models based on observed response patterns (e.g., Patz and Junker 1999a, b) or sum scores (Johnson 2013). However, these algorithms are designed to simulate realizations from the posterior distribution of the item parameters and latent traits given a sample of response patterns or sum scores, whereas here the problem is one of simulating realizations of the latent traits given a particular response pattern or sum score and known (or estimated) item parameters. In such cases, it is much more efficient to sample directly from the conditional posterior distribution of the latent trait given the response pattern or sum score, rather than from the joint distribution of all the latent traits and item parameters. ${ }^{1}$ This more direct approach is also easier to extend. The Itbayes package for $\mathrm{R}$ was developed to do just this and to provide several other tools to investigate the posterior distributions of latent traits of item response models.

The ltbayes package includes two classes of functions. One is a set of functions that evaluate unnormalized posterior distributions of the form Eq. 5, with Eq. 1 as a special case. Functions for a variety of binary and polytomous item response models are included. For binary responses, these include the three-parameter logistic model (Birnbaum 1968) and the four-parameter logistic model (Barton and Lord 1981), as well as the two-parameter and one-parameter models as special cases, and probit versions of all of these models. Functions are also available for several polytomous models, including the logistic and probit versions of the graded response model (Samejima 1969, 1972), the rat-

\footnotetext{
${ }^{1}$ Interestingly, the problem of describing the distribution of $\zeta$ given an observed sum score was also discussed by Lord $(1953,1980)$. He described a simple but inefficient process whereby response patterns are sampled for each value of $\zeta$ from a (uniform) grid of values, and then the distribution of $\zeta$ is approximated from the empirical distribution of $\zeta$ given each sum score. This provides an approximation of the posterior distribution of $\zeta$ given $\tilde{Y}=\tilde{y}$, assuming a discrete uniform prior distribution for $\zeta$.
}

ing scale model (Andersen 1977; Andrich, 1978a, b), the partial credit model (Masters 1982), the sequential model (Tutz 1990, 1997; Verhelst et al. 1997), and the nominal response model (Bock 1972). These functions can also be used for special cases of all of these models such as Muraki's (1990) rating-scale model as a special case of the graded response model. These functions use dynamically loaded shared objects that are compiled from Fortran 90/95 source code for higher computational efficiency for the utility programs discussed below, particularly when conditioning on a potentially large set of response patterns. Userspecified item response models and/or priors can also be used.

The second class of functions is used in conjunction with the first described above to investigate the posterior distribution for a given model and observed response pattern or set of response patterns. The primary function for this generates simulated realizations from the posterior distribution using a random-walk Metropolis algorithm (Metropolis et al. 1953) by leveraging the mcmc package (Geyer and Johnson 2013) and the MHadaptive (Chivers 2012) package, although it could be modified to use other routines. The simulated realizations can then be processed directly or by using one of several $\mathrm{R}$ packages for processing MCMC output such as CODA (Plummer et al. 2006), ggmcmc (Fernández 2013), or mcmcplots (Curtis 2012). These packages are useful for summarizing output as well as obtaining diagnostics to assess mixing and convergence of the chain. Additional utility functions are also included that derive various properties of the posterior distributions or likelihood functions of latent traits. These include functions to compute posterior modes, compute Fisher information (test, item, and category) and observed information, profile the posterior distribution or the (log)likelihood function, and compute profile likelihood confidence intervals.

The following sections illustrate the capabilities of the Itbayes package. These and other examples are provided with the documentation included with the package.

\section{Posterior distributions}

Consider a binary three-parameter logistic model

$$
P\left(Y_{j}=y \mid \zeta, \eta\right)=y \gamma_{j}+\frac{\left(1-\gamma_{j}\right) \exp \left[y \alpha_{j}\left(\zeta-\beta_{j}\right)\right]}{1+\exp \left[\alpha_{j}\left(\zeta-\beta_{j}\right)\right]}
$$

where $y=0,1$ and $\alpha_{j}, \beta_{j}$, and $\gamma_{j}$ are the discrimination, difficulty, and guessing parameters, respectively, with a standard normal prior $\zeta \sim N(0,1)$. Using an example from Hambleton et al. (1991), consider a $m=5$ item test with the item parameters shown in Table 1. A sample of 5,000 realizations from the posterior distribution given the 
Table 1 Item parameters for five items of a binary three-parameter logistic model

\begin{tabular}{llllll}
\hline & \multicolumn{1}{l}{ Item } & & & & \\
\cline { 2 - 6 } Parameter & $j=1$ & $j=2$ & $j=3$ & $j=4$ & $j=5$ \\
\hline discrimination $\left(\alpha_{j}\right)$ & 1.27 & 1.34 & 1.14 & 1.00 & 0.67 \\
difficulty $\left(\beta_{j}\right)$ & 1.19 & 0.59 & 0.15 & -0.59 & -2.00 \\
guessing $\left(\gamma_{j}\right)$ & 0.10 & 0.15 & 0.15 & 0.20 & 0.10 \\
\hline
\end{tabular}

response pattern $\boldsymbol{y}_{i}^{\prime}=(0,0,1,1,1)$ after a burn-in of 1,000 samples can be obtained as follows.

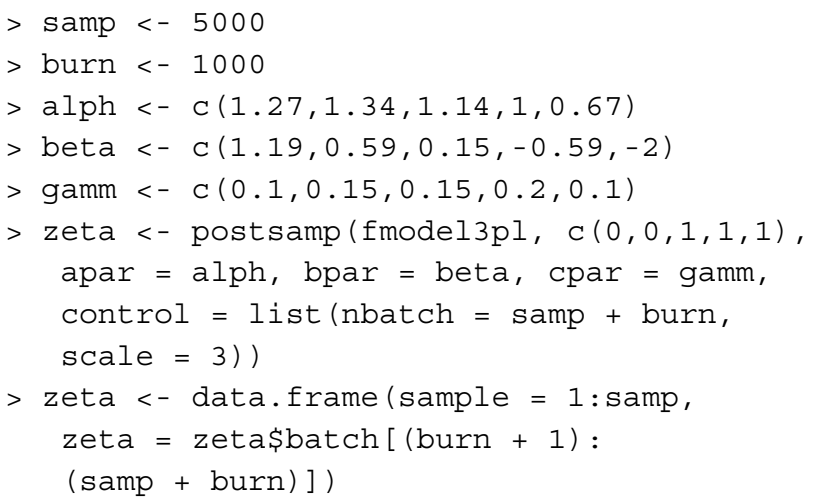

Here, post samp is the function that executes the sampling from the posterior for this model defined by the function fmodel3pl, both of which are included in the ltbayes package. The first argument of postsamp is the function that returns the log-posterior of the model, and the second argument is the response pattern. The other arguments pass the item parameters to fmodel3pl (apar, bpar, and cpar) and options to the sampling algorithm such as the number of samples (nbatch) and a scale factor for the proposal distribution (scale). A scale factor of three was specified to improve the mixing after observing the trace plot using the default value of one. The default prior distribution for $\zeta$ is $N(0,1)$, but this can also be changed using an optional argument as shown in a later example. Figure 1 shows a trace plot and posterior density estimate based on the simulated realizations stored in zeta. Figure 1a can be used to examine the performance of the sampling algorithm, whereas Fig. 1b is an estimate of the posterior distribution of $\zeta$ given the specified response pattern. Figure 2 shows the posterior distribution of $\zeta$ for each of the $2^{5}=32$ possible response patterns, including that shown in Fig. 1b, organized by sum score.

The figure illustrates how the posterior distribution of $\zeta$ depends on the response pattern.

Now consider the conditional distribution of $\zeta$ given an observed sum score $\tilde{y}=\sum_{j=1}^{m} y_{j}$. Realizations from this posterior distribution can also be generated using the postsamp function. Its second argument can be an item response pattern, or a matrix of $s$ item response patterns collected into a $s$ by $m$ matrix. The function patterns is included with the ltbayes package to generate this matrix. In general, patterns ( $m, r$, total) will generate the response patterns for $\mathrm{m}$ items, each with $r$ response categories, where the sum score is included in the (a)

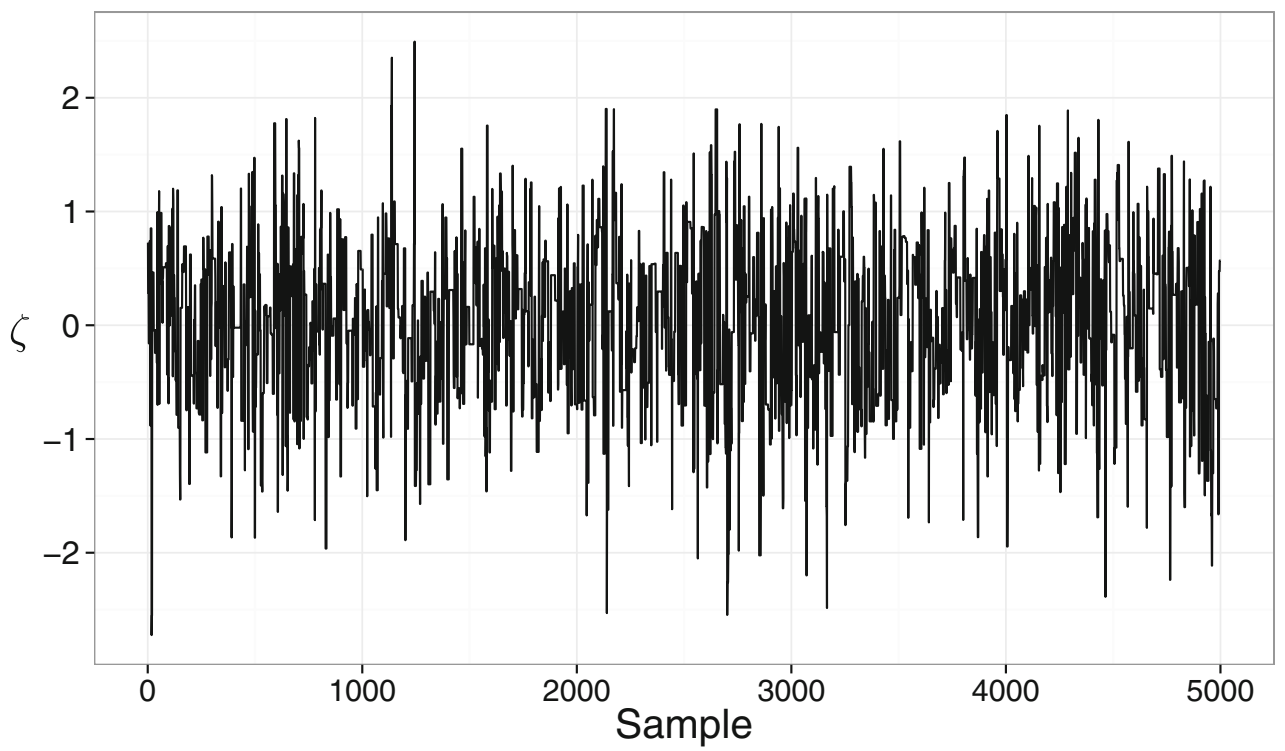

(b)

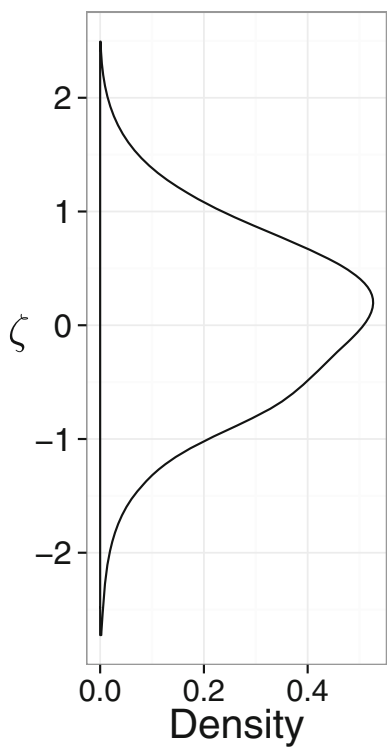

Fig. 1 Trace plot (a) and estimated posterior density (b) for $\zeta$ based on 5,000 simulated realizations from the posterior distribution 


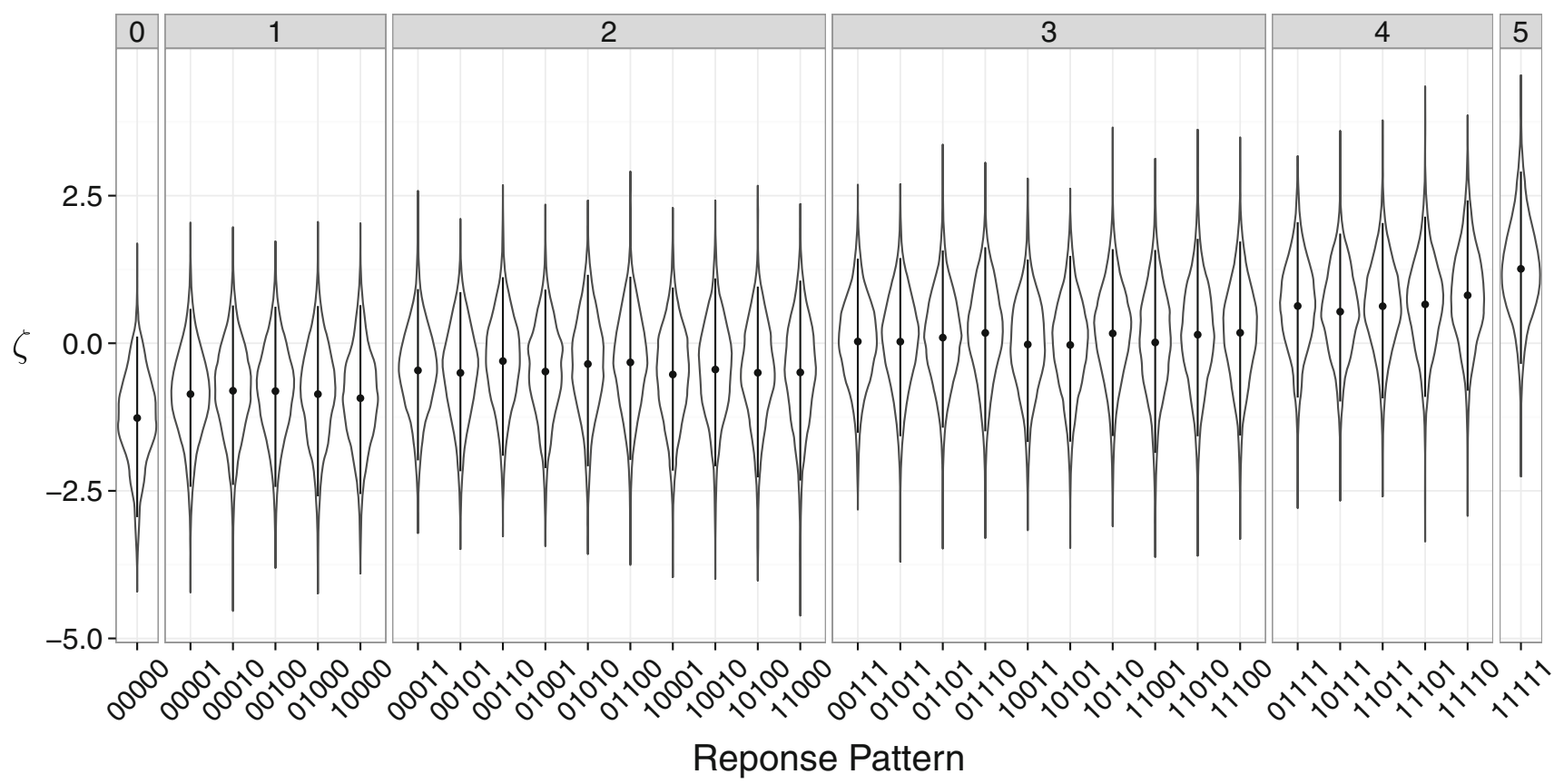

Fig. 2 Violin plots for 5,000 simulated realizations from the posterior distribution of $\zeta$ given each of 32 possible response patterns, stratified by sum score. Points and line segments within each violin plot represent the posterior mean and $95 \%$ credibility interval, respectively

vector total. For example, realizations from the posterior distribution given $\tilde{Y}_{j}=3$ can be generated as follows.

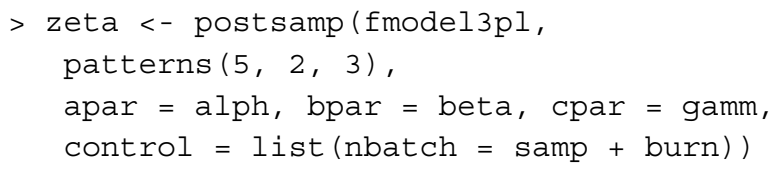

(a)

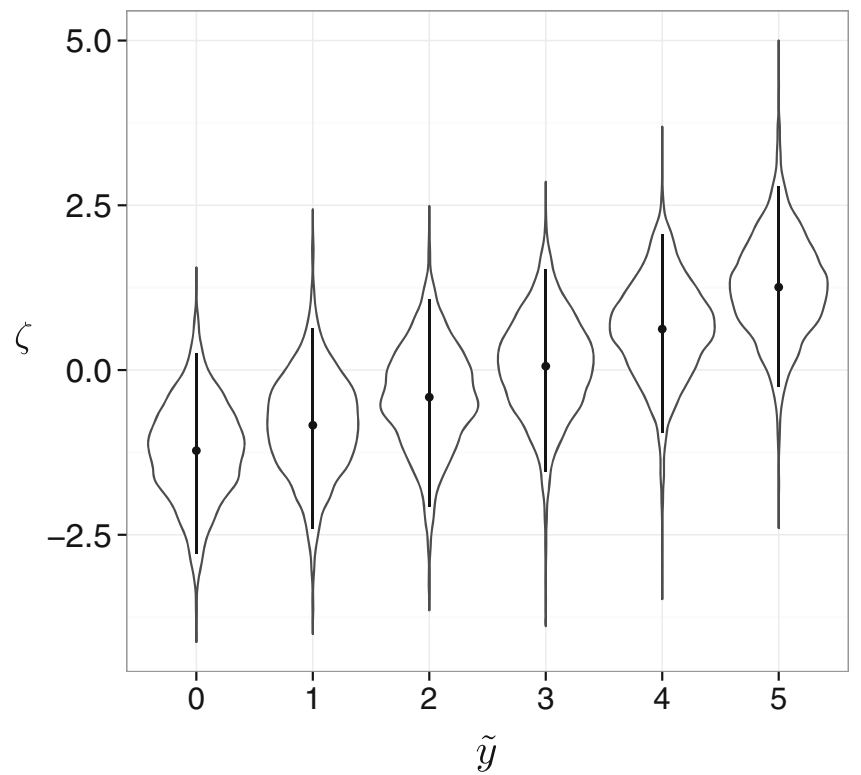

Here, patterns $(5,2,3)$ generates all response patterns for $m=5$ items with $r=2$ response categories each that yield a sum score of $\tilde{y}=3$. Figure 3 a shows the posterior distributions of $\zeta$ given each possible sum score (i.e., $\tilde{Y}=0,1, \ldots, 5$ ). Note that this figure is analogous to Fig. 2 except here the posteriors are conditional on sum scores rather than response patterns. With the exception of

(b)

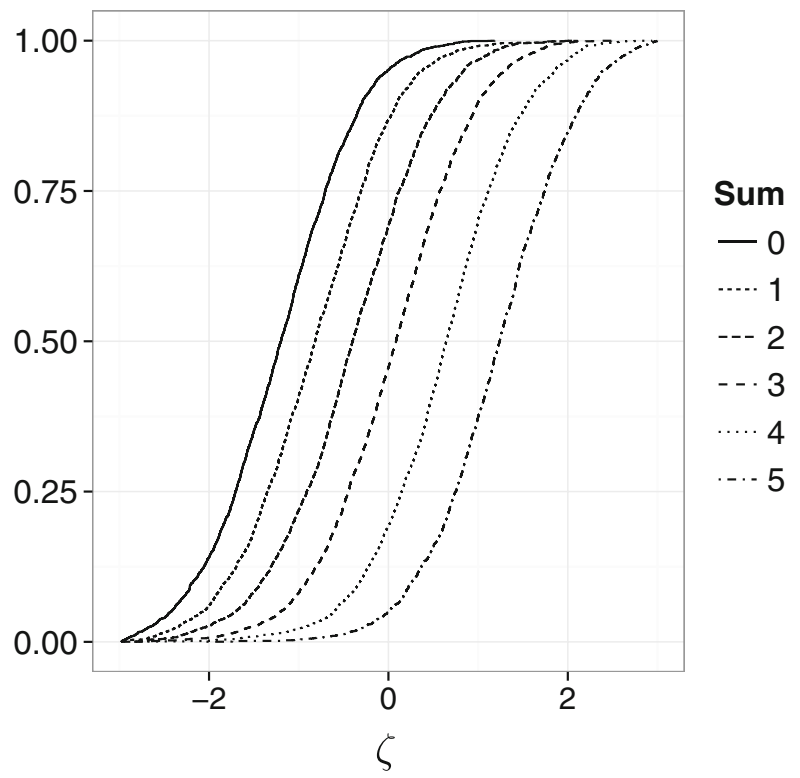

Fig. 3 Violin plots (a) and empirical cumulative distribution functions (b) for 5,000 simulated realizations from the posterior distribution of $\zeta$ conditional on sum score. Points and line segments within each violin plot represent the posterior mean and $95 \%$ credibility interval, respectively 


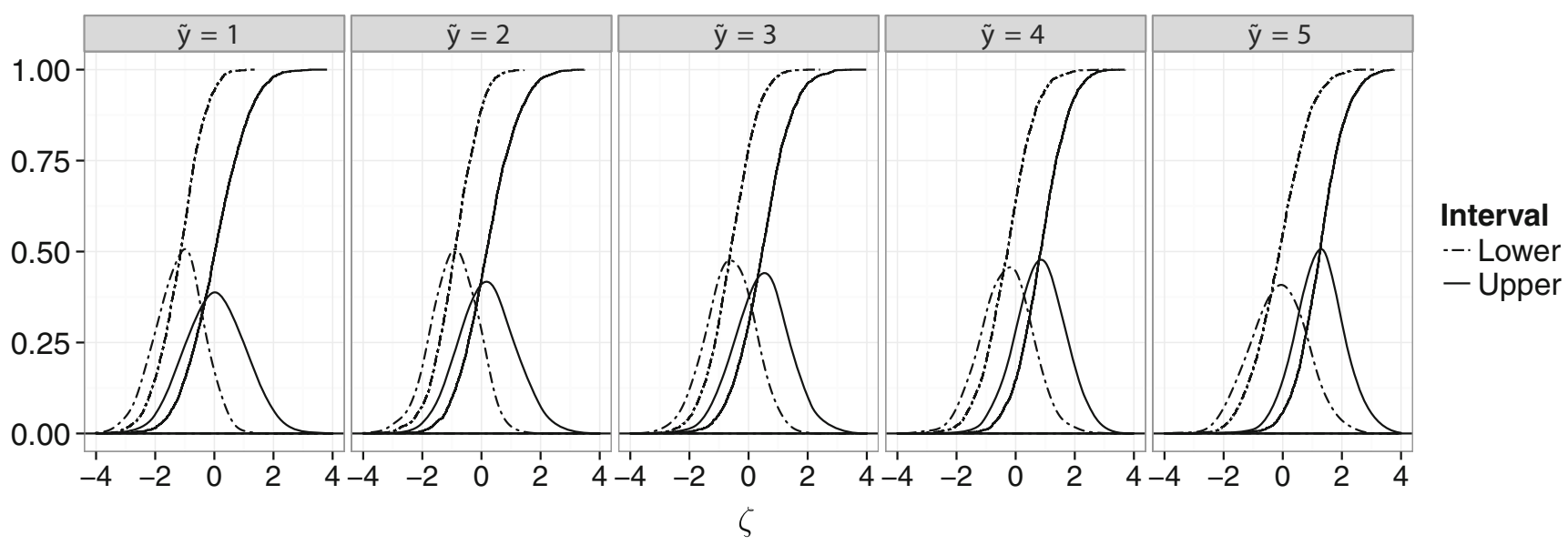

Fig. 4 Estimated density functions and empirical cumulative distribution functions for the posterior distributions of $\zeta$ given $\tilde{Y}<\tilde{y}$ (lower interval) versus $\tilde{Y} \geq \tilde{y}$ (upper interval) for $c=1,2, \ldots, 5$

the case where $\tilde{Y}=0$ or $\tilde{Y}=5$, there will be some loss of information, but in some cases like this one this may be negligible. In Fig. 2, the differences among the response patterns are much more pronounced between sum scores than within sum scores.

Note that the posterior means shown in Fig. 3 are increasing with $\tilde{y}$. This is guaranteed by the stochastic ordering of $\zeta$ by $\tilde{Y}$. The cumulative distribution functions shown in Fig. $3 b$ show the stochastic ordering of the posterior distributions of $\zeta$ given $\tilde{Y}$. Weak stochastic ordering can be seen by considering the posterior distributions of $\zeta$ given $\tilde{Y} \geq \tilde{y}$ versus $\tilde{Y}<\tilde{y}$ as shown in Fig. 4. Recall that weak stochastic ordering implies that respondents can be meaningfully categorized as "high" and "low" in terms of their latent traits after dichotomizing them with respect to their sum scores. The bell-shaped curves in each panel of Fig. 4 depict the posterior distributions of the latent traits of these two groups.

The simulated realizations of $\zeta$ can also be used to construct simple posterior prediction probabilities. This could

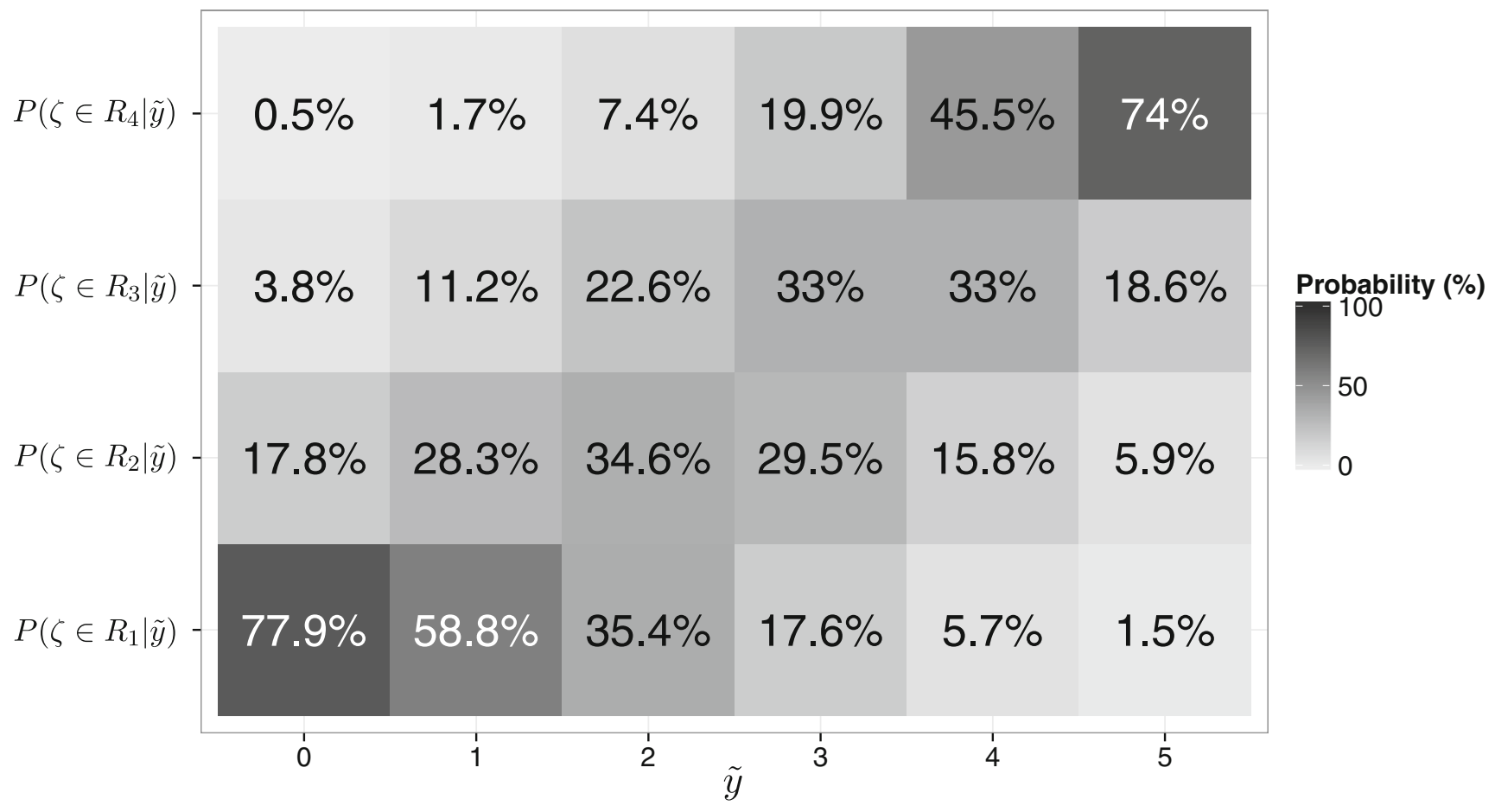

Fig. 5 Posterior probabilities, conditional on sum score $(\tilde{y})$, of $\zeta$ falling into one of four consecutive intervals defined as $R_{1}=\left\{\zeta \mid \zeta<Q_{1}\right\}$, $R_{2}=\left\{\zeta \mid Q_{1}<\zeta<Q_{2}\right\}, R_{3}=\left\{\zeta \mid Q_{2}<\zeta<Q_{3}\right\}$, and $R_{4}=\left\{\zeta \mid \zeta>Q_{4}\right\}$ where $Q_{1}, Q_{2}$, and $Q_{3}$ are the quartiles of the prior distribution of $\zeta$ 
be used to illustrate the relationship between sum and latent traits for laypersons by creating simple prediction rules based on sum scores. For example, consider the posterior probability that $\zeta$ falls into some range of values $R$ given $\tilde{Y}=\tilde{y}$. These posterior probabilities can be trivially computed using the output from post samp by tabulating zeta. Figure 5 illustrates the posterior probabilities of $\zeta$ being within one of the four quartiles of the prior distribution of $\zeta$. This shows very simply the probability, given the observed sum score, that a respondent can be placed in a given quartile. Another example is to compare two examinees based on their sum scores. Figure 6 shows the posterior probability that the latent traits of one examine is larger than that of the other given their sum scores. Posterior probabilities like these provide for easy-to-understand statistical predictions while also effectively communicating the uncertainty in those predictions. The probabilities communicate the (un)certainty concerning the relative position of a respondent, or the relative position of two respondents, given their test performance. This kind of analysis is not restricted to sum scores. Similar plots could also be constructed using response patterns, expanding the grid of cells in Fig. 5 to 4 by 32, and Fig. 6 to 32 by 32 .

Posterior profiles and profile likelihood

The posttrace function in ltbayes computes the unnormalized logarithm of the posterior density defined in Eqs. 1 or 5 for a grid of values of $\zeta$. This can be useful for studying the profile of the posterior distribution or the likelihood function and their properties such as curvature and modes. Continuing with the same example from the

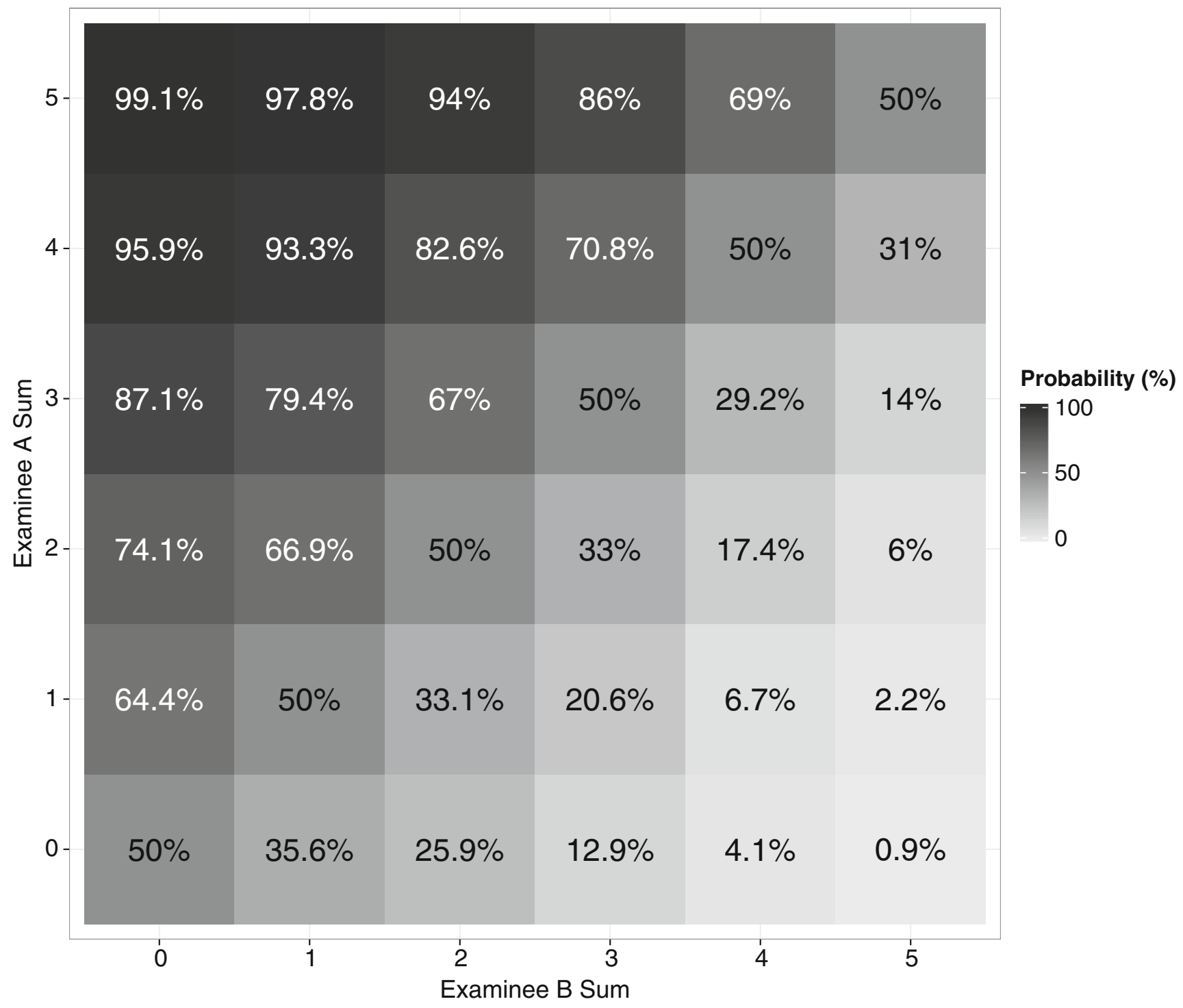

Fig. 6 Posterior probabilities of the form $P\left(\zeta_{A}>\zeta_{B} \mid \tilde{Y}_{A}=\tilde{y}_{A}, \tilde{Y}_{B}=\tilde{y}_{B}\right)$ - i.e., the posterior probability that examinee A has a larger latent trait than examinee $B$ given their sum scores 
previous section, the profile of the posterior distribution of $\zeta$ given $\tilde{Y}=3$ can be computed as follows.

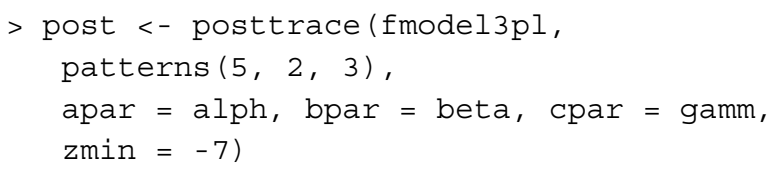

The arguments to posttrace are like those for postsamp except here we include an optimal argument zmin to extend the lower limit of the $\zeta$ from five (the default) to seven. The object post is a list of two objectsthe values of $\zeta$ and the corresponding values of the unnormalized log-posterior density in the interval $[-7,5]$. Figure 7 shows a plot of this profile and those for the other possible sum scores for a standard normal prior and an improper uniform prior $g(\zeta) \propto 1$ which is obtained by specifying prior $=$ function $(z) \quad 1$. Recall that when $g(\zeta) \propto 1$, the posterior is the likelihood function, so posttrace returns the profile log-likelihood function. Note that the posteriors with the uniform prior are not necessarily unimodal. The posterior for $\tilde{Y}=0$ and $\tilde{Y}=5$ are nonincreasing and non-decreasing, respectively (the posterior for $\tilde{Y}=1$ only appears to be non-increasing, but in fact is unimodal although this is not evident in the figure because of the scale of the ordinate).
Two additional functions included with ltbayes are useful for working with the profile posterior/likelihood function. The function postmode tries to numerically compute the mode of the posterior distribution, and profileci tries to compute the profile likelihood confidence interval for $\zeta$. Note that these functions may not be successful if the posterior is not unimodal or a closed interval cannot be found. The $\mathrm{R}$ code below demonstrates the calculation of the mode of the profile likelihood (i.e., the MLE of $\zeta$ ) and the profile likelihood interval for $\zeta$ given $\tilde{Y}=3$.

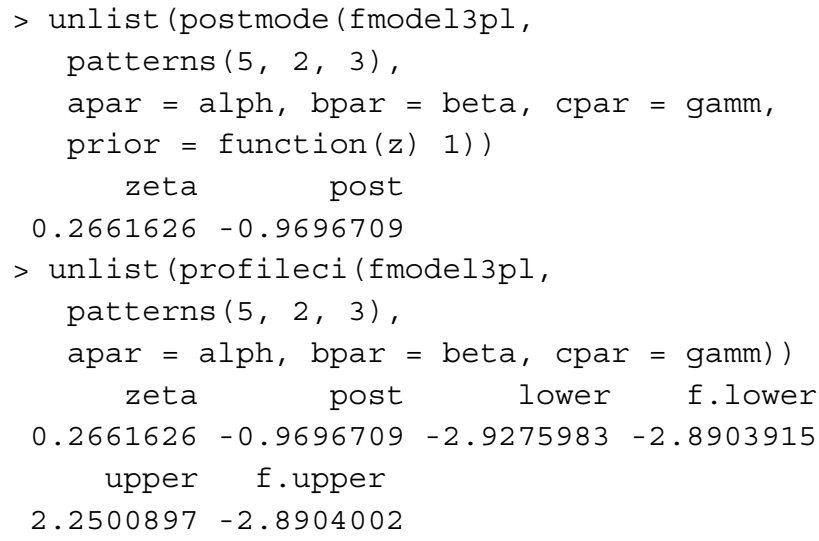

Here, the MLE of $\zeta$ is approximately 0.26 with a profile confidence interval of approximately $(-2.93,2.25)$.

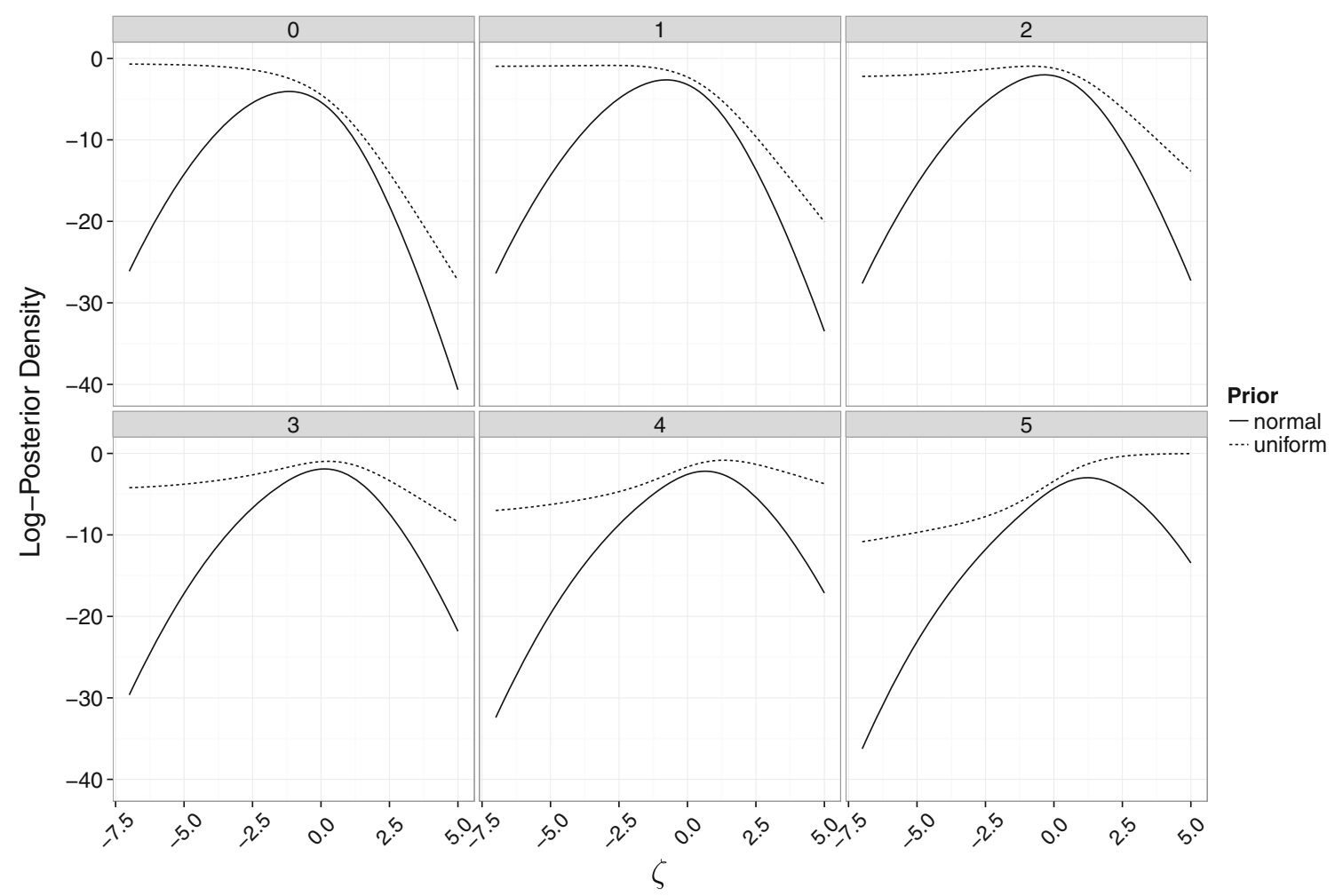

Fig. 7 Profiles of the unnormalized log-posterior distribution of $\zeta$ given $\tilde{Y}=0,1, \ldots, 5$ with normal and improper uniform prior distributions 
Figure 8 illustrates the profile log-likelihood functions when $\tilde{Y}=2,3,4$ and the profile likelihood confidence intervals when $\tilde{Y}=3,4$ (the interval is not closed when $\tilde{Y}=2$. The function postmode returns the MAP or MLE estimate of $\zeta$ depending on if the prior is uniform, but the function profileci automatically assumes a uniform prior so that the posterior is proportional to the likelihood.

While the examples above feature investigations of the posterior distribution or likelihood function given a sum score, similar analyses can be performed using posttrace, postmode, and profileci given a response pattern. As far as the functions are concerned, this is a special case where the set of response patterns is a single response pattern.

Fisher and observed information

The ltbayes package includes the function information which can compute Fisher or observed information. Using the same example from above, the test, item, and category Fisher information at the MLE of $\zeta$ can be computed as follows.

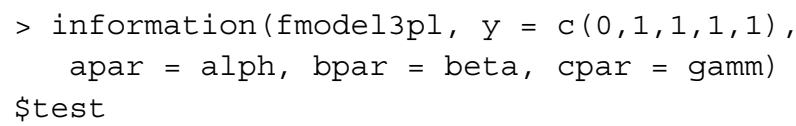

[1] 0.9327704

\$item
[1] $0.33214773 \quad 0.29216270 \quad 0.17916382$
$0.08946765 \quad 0.03982853$

\$category

$$
[, 1] \quad[, 2]
$$

$[1] \quad 0.169867871 \quad$,

$[2] \quad 0.087441737 \quad$,

$[3] \quad 0.040131292 \quad$,

$[4] \quad 0.012258137 \quad$,

$[5] \quad 0.003973319 \quad$,

The arguments for information are like those for the functions discussed earlier. Note that Eq. 3 does not depend on the actual item responses so the same results would be obtained when replacing the $y$ argument with any other appropriate response pattern. By default, information

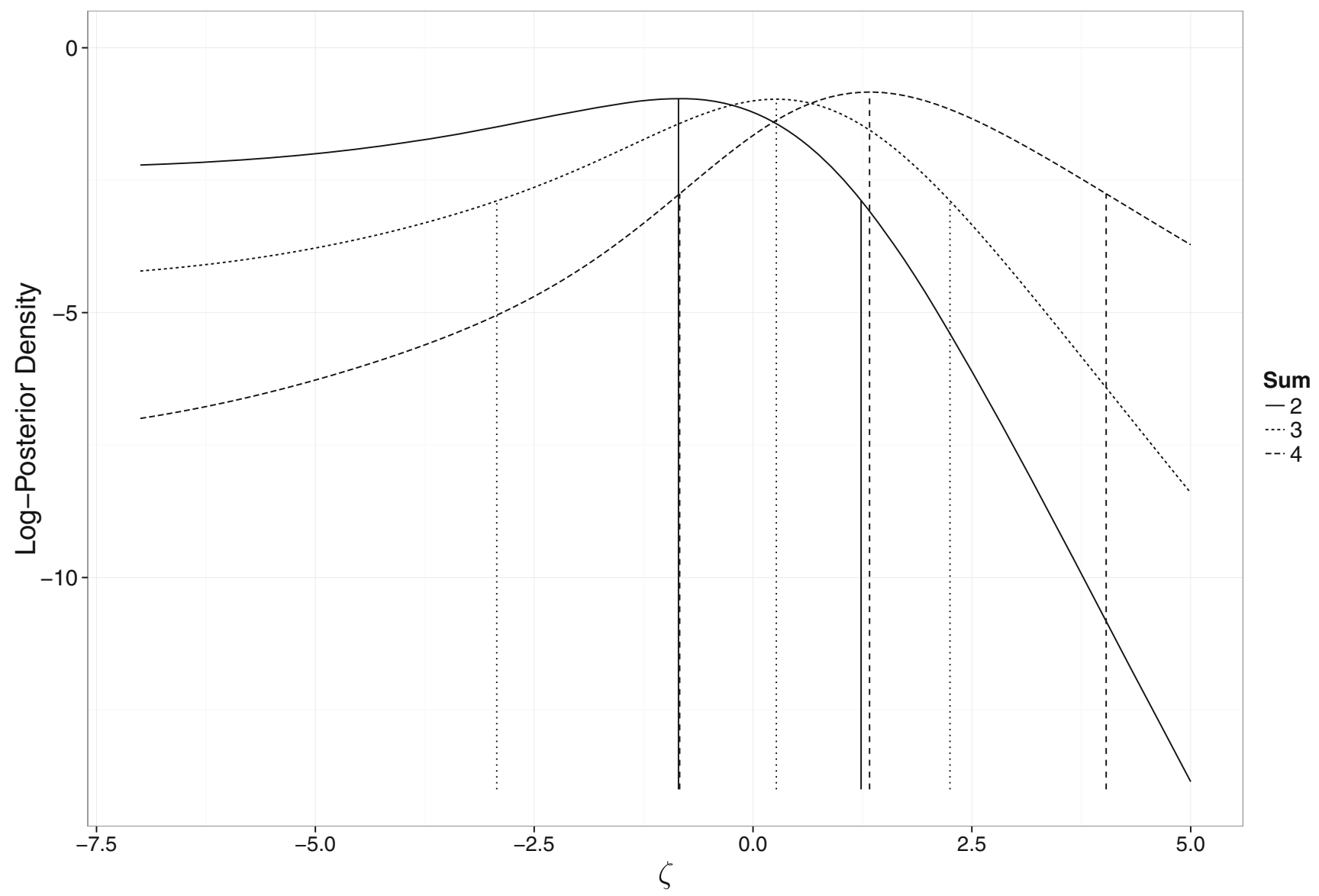

Fig. 8 Profile likelihoods for $\zeta$ given $\tilde{Y}=2,3,4$ with maximum likelihood estimates and profile likelihood confidence intervals 


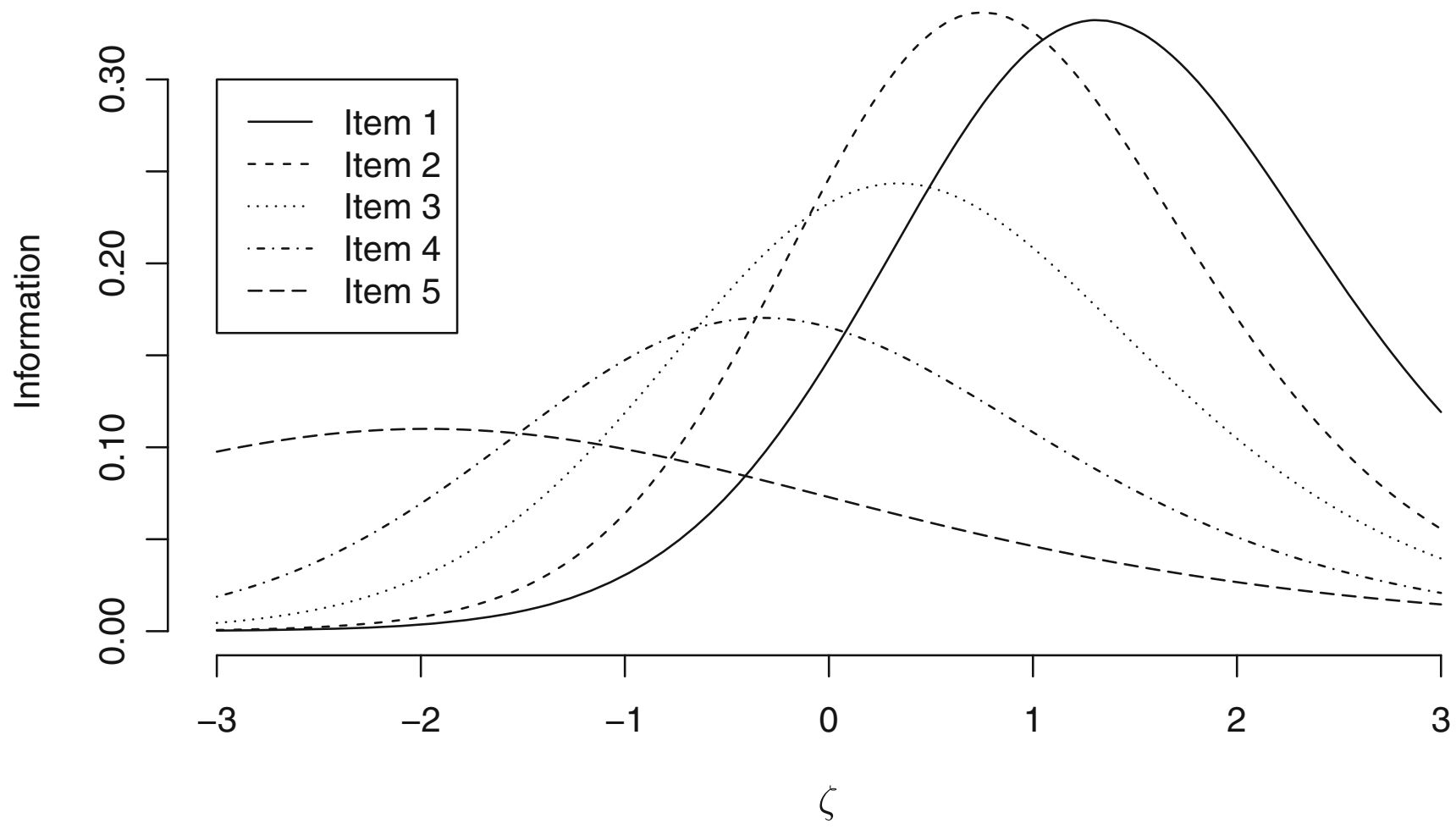

Fig. 9 Item information functions for five items of a three-parameter binary logistic model

returns the Fisher information at the maximum likelihood estimate of $\zeta$, but it can easily be used to compute the information at an arbitrary value of $\zeta$. The example below shows how to compute the item information at a grid of values of $\zeta$ for plotting.

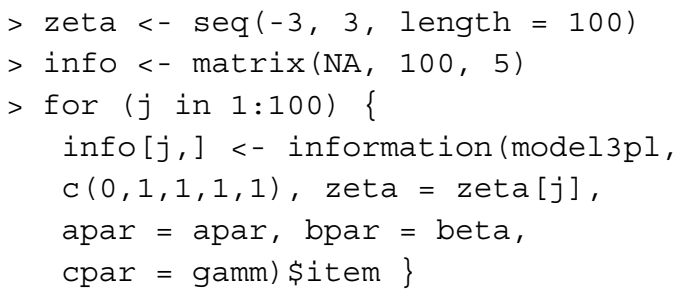

The item information functions can then be plotted using info as shown in Fig. 9.

When using a sum score instead of a response pattern as the data, the Fisher information becomes

$I(\zeta)=-E\left\{\frac{\partial^{2}}{\partial \zeta^{2}} \log \left[\sum_{\mathbf{z} \in S} \prod_{j=1}^{m} P\left(Y_{j}=z_{j} \mid \zeta, \boldsymbol{\eta}_{j}\right)\right]\right\}$.

Note that as in Eq. 5, the summation is over the set of response patterns that yield a given sum score. This Fisher information does not necessarily have a closed form unless
$S$ is a single response pattern. ${ }^{2}$ An alternative to the Fisher information is the observed information, defined here as

$\tilde{I}\left(\hat{\zeta}_{S}\right)=-\left.\frac{\partial^{2}}{\partial \zeta^{2}} \log \left[\sum_{\mathbf{z} \in S} \prod_{j=1}^{m} P\left(Y_{j}=z_{j} \mid \zeta, \boldsymbol{\eta}_{j}\right)\right]\right|_{\zeta=\hat{\zeta}_{S}}$

where $\hat{\zeta}_{S}$ is the MLE of $\zeta$ given that $\boldsymbol{y} \in S$. Unlike Fisher information, observed information is only meaningfully defined at the maximum likelihood estimator $\hat{\zeta}_{i}$. For some models, such as the one- and two-parameter binary logistic models, the Fisher and observed information in Eq. 6 are equal at $\hat{\zeta}_{S}$ if $S$ is a single response pattern. They are not necessarily equal in general but should be similar for models with a large number of items. By default, information computes the Fisher information if $S$ is a single response pattern, but the observed information can be obtained using the observed = TRUE option.

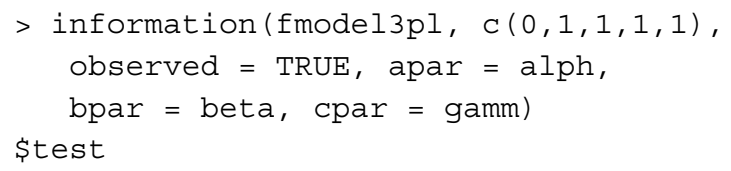

[1] 0.9675214

${ }^{2}$ In principle, the Fisher information could be approximated numerically using a simulation-based method, but this is currently beyond the capabilities of ltbayes. 
In the case of the three-parameter binary logistic model, the Fisher and observed information are not necessarily equal at $\hat{\zeta}_{S}$. The main advantage of the observed information is for computing the information and the standard error when estimating $\zeta$ conditional on a set of response patterns such as a sum score. For example, the observed information for a sum score of $\tilde{y}=4$ is obtained as follows.

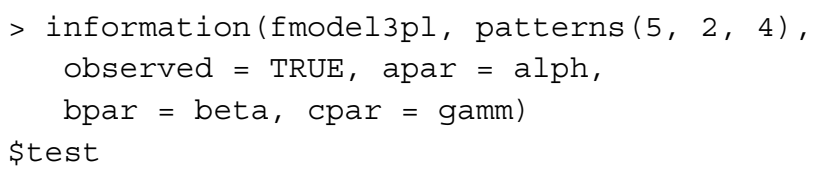

[1] 0.8992739

Note that the observed information is somewhat lower here because conditioning on the sum score is not as efficient as conditioning on the response pattern, although perhaps not as low as one might expect.

\section{User-defined models}

One major feature of ltbayes is that its functions will also work with user-specified models. Users can specify their own item response function of the form $P\left(Y_{i j}=y \mid \zeta, \boldsymbol{\eta}_{j}\right)$ and/or a posterior distribution of the form $g(\zeta \mid \omega)$. They are not limited to the models that are included with the package. For an example, consider the hyperbolic cosine unfolding model for binary responses (Andrich and Luo 1993). The item response function for this model can be written as

$$
P\left(Y_{j}=y \mid \zeta, \boldsymbol{\eta}\right)= \begin{cases}2 \cosh \left(\zeta-\beta_{j}\right) / c_{j}, & \text { if } y=0, \\ e^{\alpha_{j}} / c_{j}, & \text { if } y=1,\end{cases}
$$

for $y=0,1$ where $\zeta$ and $\beta_{j}$ are the respondent and item location parameters, respectively, $\alpha_{j}$ is a "unit" parameter, and $c_{j}$ is the normalizing constant (i.e., the sum of the numerators of the terms when $y=0$ and $y=1$ ). Figure 10 shows the item response probabilities for a hyperbolic cosine model with $\alpha_{j}=1$ and $\beta_{1}=-2, \beta_{2}=-1$, $\beta_{3}=0, \beta_{4}=1$, and $\beta_{5}=2$. Unlike many item response models, the item response function of the hyperbolic cosine model is not monotonic but instead is single-peaked as is typical for unfolding models. The peak of each symmetric function is equal to the corresponding value of $\beta_{j}$, and the height and shape depend on $\alpha_{j}$. To implement this model with Itbayes, what is needed is a function to evaluate its posterior distribution, such as the following.

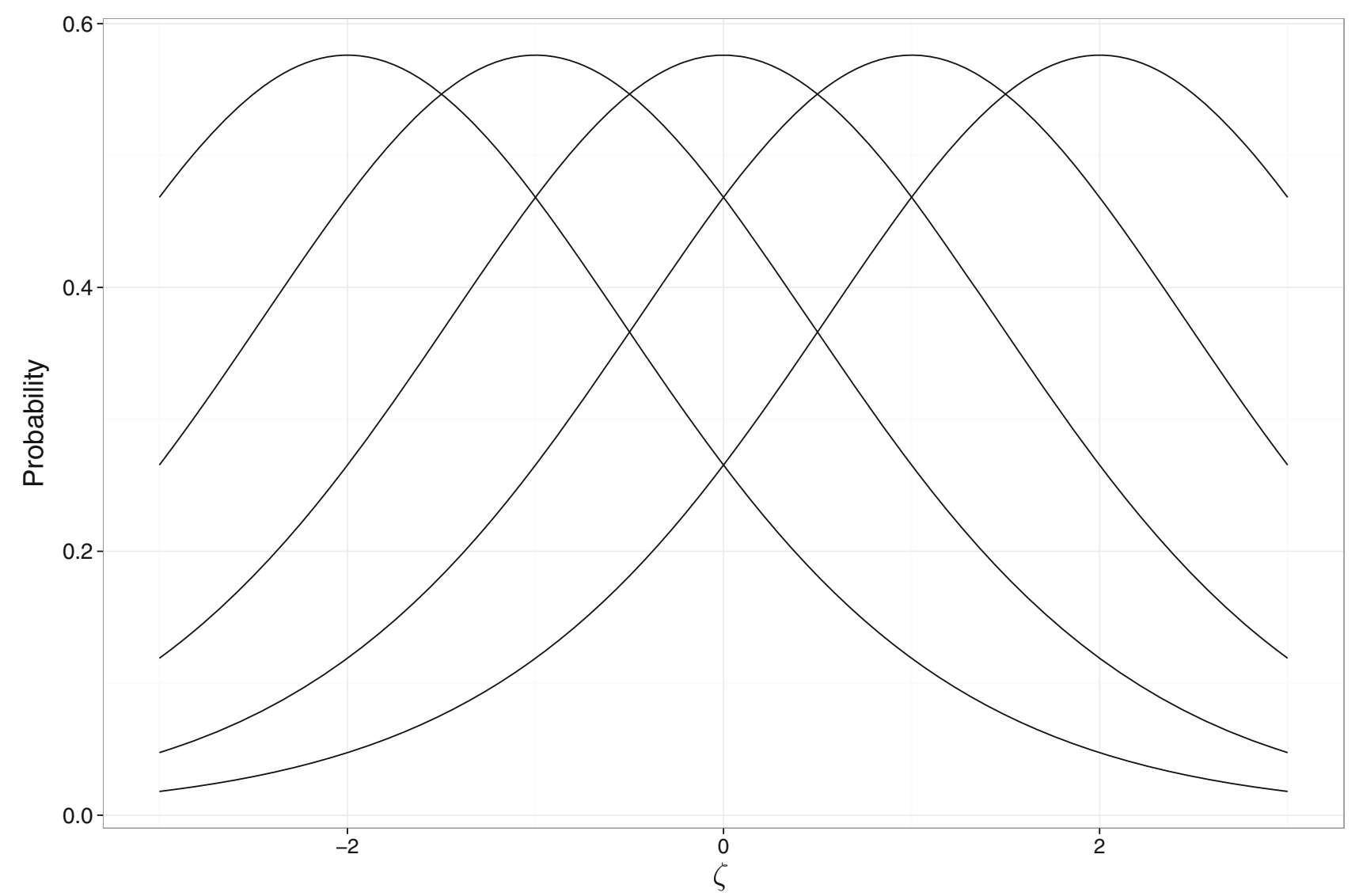

Fig. 10 Item response probabilities for a $m=5$ item hyperbolic cosine model with item parameters $\alpha_{j}=1$ and $\beta_{1}=-2, \beta_{2}=-1, \beta_{3}=0$, $\beta_{4}=1$, and $\beta_{5}=2$ 


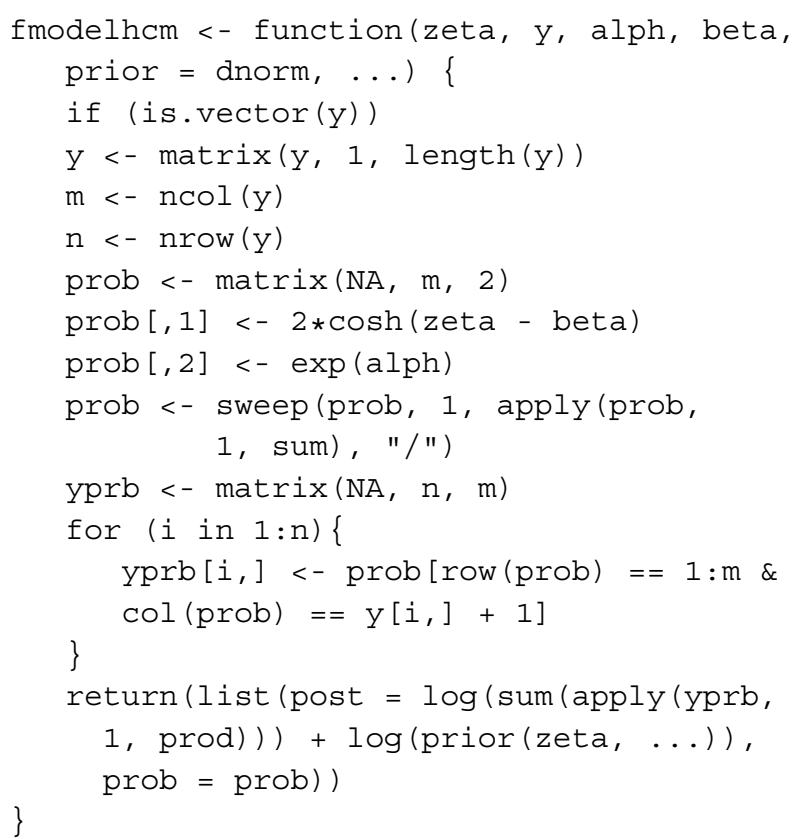

The utility functions in Itbayes expect that the first argument is $\zeta$, and that the function returns a list of the $\log$-posterior at $\zeta$ named post, and for use with information a $m$ by $r$ matrix of category probabilities for each item named prob. The second argument is the $s$ by $m$ matrix of item response patterns where $s$ is the number of elements in $S$ in Eq. 5. This includes the case where $S$ is a single response pattern. So the first two arguments of a custom function like fmodel hcm must correspond to the latent trait and the response vector/patterns. After that additional arguments can be used to pass the item/category parameters, prior distribution, and any other values. The ellipsis "argument" allows the user to pass arguments to the function defining the prior distribution if necessary. Some readers may see ways to make this function more efficient using $\mathrm{R}$ and/or subroutines written in $\mathrm{C}$ or Fortran, but for many purposes a simple $\mathrm{R}$ function may be quite sufficient. The function assumes a standard normal prior distribution (dnorm) by default, but here we will consider a two-component normal mixture distribution such that the prior distribution of $\zeta$ is

$\pi \phi\left(\zeta, \mu_{1}, \sigma_{1}\right)+(1-\pi) \phi\left(\zeta, \mu_{2}, \sigma_{2}\right)$

where $0 \leq \pi \leq 1$ is the mixing parameter and $\phi$ denotes the probability density function of a normal distribution with

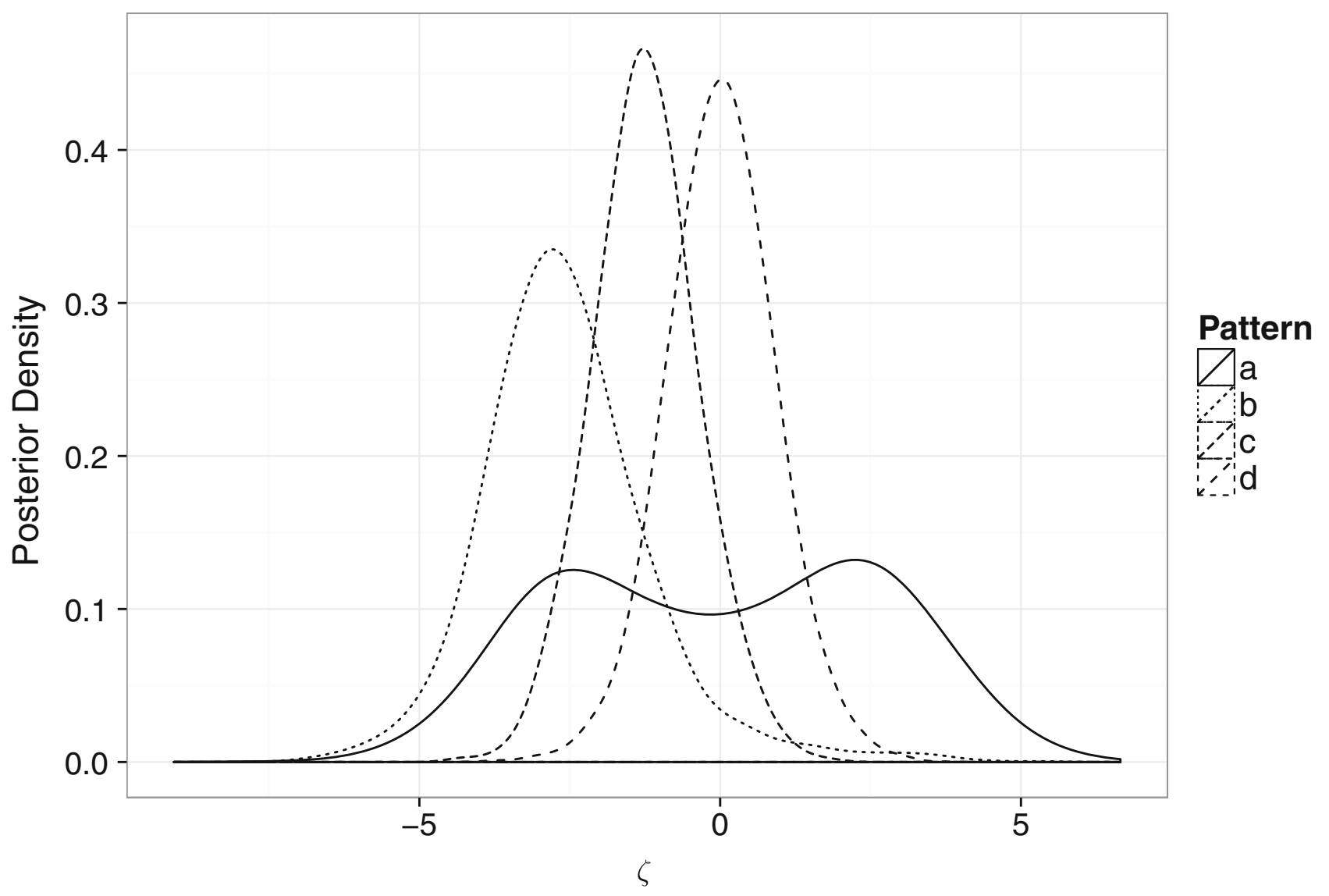

Fig. 11 Posterior distribution of $\zeta$ for the hyperbolic cosine model given $\tilde{y}=1(\mathbf{a}), \boldsymbol{y}^{\prime}=(1,0,0,0,0)(\mathbf{b}), \boldsymbol{y}^{\prime}=(1,1,1,0,0)(\mathbf{c})$, and $\boldsymbol{y}^{\prime}=(0,1,1,1,0)(\mathbf{d})$ 
given mean and standard deviation. All that is needed for this prior is a function that returns this probability density with a first argument of $\zeta$.

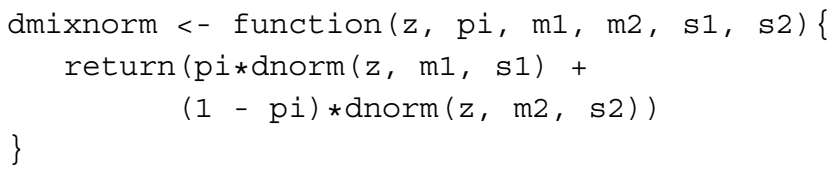

Now, using fmodelhem and dmixnorm, the hyperbolic cosine model with a two-component normal mixture prior for $\zeta$ can be investigated using the Itbayes package.

Here we consider a $m=5$ item model with item parameters defined earlier with response functions shown in Fig. 10. The prior for $\zeta$ is a two-component normal mixture with $\mu_{1}=-2, \mu_{2}=2, \sigma_{1}=\sigma_{2}=2$, and $\pi=0.5$. A sample of 10,000 realizations from the posterior distribution of $\zeta$ for a given response vector $\mathrm{y}$ can be generated as follows.

$>$ zeta <- postsamp (fmodelhcm, y,

alph $=\operatorname{rep}(1,5)$, beta $=-2: 2$,

prior = dmixnorm,

control $=$ list $($ nbatch $=10000)$,

$\mathrm{m} 1=-2, \mathrm{~m} 2=2, \mathrm{~s} 1=2, \mathrm{~s} 2=2$,

$\mathrm{pi}=0.5)$
Note that the function that defines the posterior distribution is passed to postsamp as prior along with its hyperparameters. Figure 11 shows the density estimates of the posterior distribution of $\zeta$ for this hyperbolic cosine model conditional on three response vectors and a sum score of $\tilde{Y}=1$. Note the bimodality of the posterior of the sum score. A respondent who endorses only one of the five items is more likely to be relatively low or high on the scale of the latent trait. Stochastic ordering does not necessarily hold for the hyperbolic cosine model. Proofs for MLR and SOL assume monotonic (cumulative) item response functions.

Figure 12 shows the item and test Fisher information functions for the hyperbolic cosine model computed using the function information in a way similar to the threeparameter logistic model earlier. Note the bimodality of the information functions. This is an interesting characteristic of ideal point models (e.g., Verkulien 2006).

\section{Discussion}

Item response models can be used in two complementary modes of inference. One is in the scaling of items. This is useful for the purpose of test development and in studying

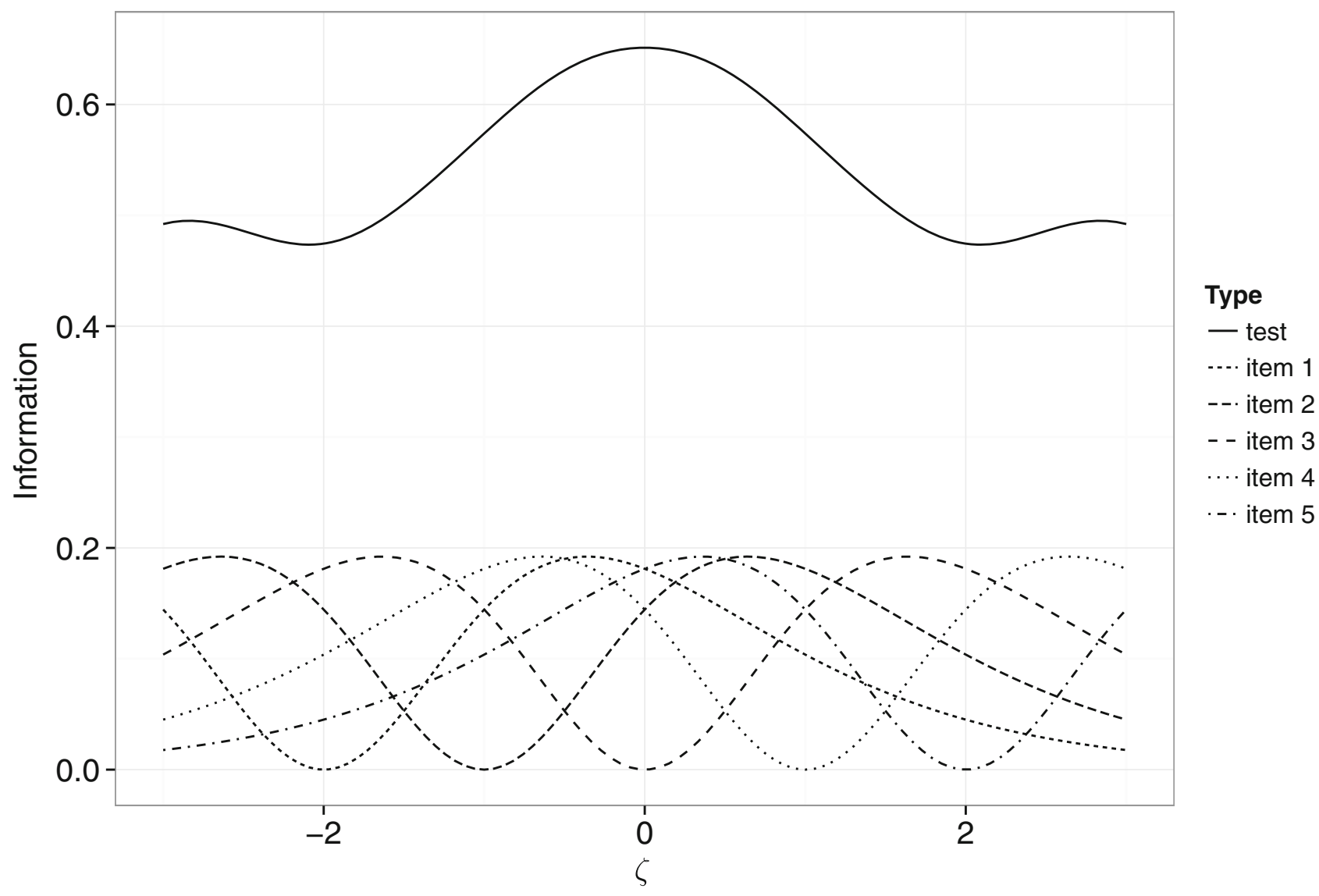

Fig. 12 Item and test Fisher information functions for five items of a hyperbolic cosine model 
how response distributions are related to properties of the items. The other is in the scaling of respondents. Here the focus is on using observed item responses to a given set of items to make inferences concerning individual respondents. Many software packages exist for the first mode of investigation, including several general-purpose packages that have been developed for $\mathrm{R}$ such as the $\mathbf{e R m}$ package (Mair and Hatzinger 2007; Mair et al. 2014) for Rasch models using conditional maximum likelihood, the Itm package (Rizopoulos 2006) for marginal maximum likelihood, and MCMCpack (Martin et al. 2011) for a Bayesian approach. The purpose of the ltbayes package is to provide a general-purpose resource for researchers and methodologists for the second mode of inference-inferences for a latent trait for a given item response model. The packages cited above and a few others such as irtProb (Raiche 2014) also include functions for latent trait estimation, but these are less flexible than the general approach used by ltbayes to derive analytically or by simulation properties of latent trait posterior distributions or likelihoods.

Another significant motivation for the development of the Itbayes package is to provide a tool for making inferences concerning latent traits accessible not just for use by researchers and methodologists but also to help them make inferences accessible to practitioners. The strong statistical foundation of item response theory is its greatest strength, but it has perhaps somewhat hindered its understanding and adoption by laypersons. We believe that by using sum scores and/or (graphical) summaries of posterior distributions, as shown in some of the examples, the capabilities and potential of item response theory for making inferences about respondents can be made more apparent. Inferences for latent traits conditioning on sum scores is also useful for understanding what happens when one chooses or is forced to rely on sum scores for scaling respondents. The ltbayes package is particularly well suited for these applications.

As one example, the spread of computerized adaptive testing and the increasing emphasis on non-cognitive assessments have highlighted the benefits of item response theory for improving personnel selection (e.g., Donovan et al. 2000; Drasgow and Hulin 1990; Schneider et al. 2003; Zickar et al. 2004). Yet there remains substantial resistance on the part of many practitioners to using validated selection aids, and to discussions of the uncertainty inherent in test data and validation analyses (Highhouse 2008). Even before the development of item response theory, researchers saw the need for user-friendly ways to express the value of selection tests. Lawshe and Bolda (1958) argued that managers could not understand validity coefficients, and developed expectancy charts showing the percentage of test takers in each score quintile who met a standard of success. Guion (2011) argued for careful and systematic training of users of selection test information, with "the most important objective [being] to develop a habit of thinking in terms of probabilities rather than certainties and of gradual changes in those probabilities rather than sudden discontinuities" (p. 424).

Given that users are often uncomfortable even with concepts from classical test theory, such as simple sums scores, then the challenges associated with getting them to accept the more abstruse counterpart of a latent trait of an item response model can be formidable. Using sum scores to infer latent traits helps to make item response theory results more easily understood. Stochastic and weak stochastic ordering imply that it is reasonable to rank or group testtakers using sum scores. Furthermore, a Bayesian approach based on posterior distributions is particularly well-suited for communicating inferences concerning respondents without requiring an understanding of the probability model itself. This can be used to create input for decisions under uncertainty. Examples of this were illustrated earlier, such as the posterior probability that a respondent's latent trait falls within a certain range (e.g., What is the probability that the examinee's aptitude is in the top quartile given their test score?), or the posterior probability of the order of two respondents with respect to their latent traits (e.g., What is the probability that one examinee's aptitude is greater than another's given their test scores?). The ltbayes package can facilitate the generation of this kind of information.

\section{Appendix: Item response models}

The Itbayes package can be applied to a wide variety of item response models for discrete item responses. These include models for binary and polytomous (ordered or unordered) responses. Some common models are briefly reviewed below, and the appropriate functions from the Itbayes package for implementing these models are noted.

\section{Binary item response models}

A general model that includes many binary item response models is

$P\left(Y_{i j}=1 \mid \zeta_{i}, \boldsymbol{\eta}\right)=\gamma_{j}+\left(\delta_{j}-\gamma_{j}\right) F\left[\alpha_{j}\left(\zeta_{i}-\beta_{j}\right)\right]$,

where $\alpha_{j}$ and $\beta_{j}$ are the "discrimination" and "difficulty parameters," respectively, and $\gamma_{j}$ and $\delta_{j}$ are the lower and upper asymptotes of the function as $\zeta_{i} \rightarrow-\infty$ and $\zeta_{i} \rightarrow-\infty$, respectively, and $F$ is a distribution function. Logistic and probit models specify that $F$ is the distribution function of a standard logistic and normal distribution, respectively. Common special cases of this four-parameter model include three-parameter models with all $\delta_{j}=1$ so that the upper asymptote is 1 , 
two-parameter models which also include the constraint that all $\gamma_{j}=0$, and one-parameter models that further specify equal discrimination parameters such that all $\alpha_{j}=1$. Clearly, other models could be specified in terms of constraints on the item parameters and/or the distribution function $F$. The ltbayes package includes functions for evaluating the posterior distribution of the latent trait for these models. These are fmodellpl, fmodel2pl, fmodel3pl, and fmodel $4 \mathrm{pl}$ for the logistic models, and fmodel 1pp, fmodel2pp, fmodel $3 \mathrm{pp}$, and fmodel $4 \mathrm{pp}$ for the probit models.

\section{Cumulative models}

Cumulative models can be written as

$P\left(Y_{i j} \geq y \mid \zeta_{i}, \eta_{j}\right)=F\left[\alpha_{j}\left(\zeta_{i}-\beta_{j y}\right)\right]$,

for $y=1,2, \ldots, r-1$, noting that $P\left(Y_{i j} \geq 0 \mid \zeta_{i}, \eta\right)=1$ by definition, and where $F$ is a distribution function. The $\alpha_{j}$ and $\beta_{j y}$ item parameters can be viewed as the "discrimination" and "difficulty" for each item when dichotomizing the response scale into a binary response. Binary item response models with lower and upper asymptotes of 0 and 1, respectively, are also cumulative models. This family of models was first proposed by Samejima $(1969,1972)$ and are often referred to as graded response models. Muraki (1990) proposed a special case of this model for rating scales where $\beta_{j k}=\delta_{j}+\gamma_{k}$ where $\delta_{j}$ and $\gamma_{k}$ are item- and categoryspecific parameters, respectively. Common graded response models are the logistic and probit versions where $F$ is the distribution function of a standard logistic and normal distribution, respectively. The ltbayes functions for the logistic and probit graded response models are fmodelgrl and fmodelgpl, respectively.

\section{Partial credit models}

Muraki (1992) proposed the generalized partial credit model, which can be written as

$$
P\left(Y_{i j}=y \mid \zeta_{i}, \boldsymbol{\eta}_{j}\right)=\frac{\exp \left(\sum_{l=0}^{y} \alpha_{j}\left(\zeta_{i}-\beta_{j l}\right)\right)}{\sum_{k=0}^{r-1} \exp \left(\sum_{l=0}^{k} \alpha_{j}\left(\zeta_{i}-\beta_{j l}\right)\right)}
$$

for $y=0,1, \ldots, r-1$ and $\beta_{j 0}=0$. The item parameters $\alpha_{j}$ and $\beta_{j l}$ can be viewed as the discrimination and difficulty parameters, respectively, when conditioning on a response being in one of two adjacent categories. Special cases include the partial credit model (Masters 1982) where all $\alpha_{j}=1$, and the rating scale model (Andersen, 1977; Andrich, 1978a, b) where all $\alpha_{j}=1$ and $\beta_{j k}=\delta_{j}+\gamma_{k}$ where $\delta_{j}$ and $\gamma_{k}$ are item- and category-specific parameters, respectively. The fmodelpcm and fmodelrsm functions in Itbayes evaluate the posterior distribution of the latent trait for the partial credit and rating scale models, respectively. Specifying a $\mathrm{R}$ function fmodelgpc for the generalized partial credit model is illustrated in a vignette included with the package.

\section{Sequential models}

Sequential item response models can be written as

$P\left(Y_{i j}>y \mid Y_{i j} \geq y, \zeta_{i}, \eta_{j}\right)=F\left(\zeta_{i}-\beta_{j y}\right)$

for $y=0,1, \ldots, r-2$, where $\beta_{j y}$ is a difficulty parameters of a response greater than $y$ given a response of at least $y$. This model was proposed by Tutz $(1990,1997)$ and Verhelst et al. (1997). Tutz (1990) also considered a rating scale variant where $\beta_{j k}=\gamma_{j}+\delta_{k}$ where $\gamma_{j}$ and $\delta_{k}$ are item- and category-specific parameters, respectively. The fmodel seq from ltbayes is for the sequential model. The rating scale variant can be implemented through this function as a special case. The ltbayes package does not currently include a function for the more general model with unequal discrimination parameters, but one can be relatively easily programmed in $R$.

\section{Nominal response models}

The nominal response model proposed by Bock (1972) is

$$
P\left(Y_{i j}=y \mid \zeta_{i}, \eta_{j}\right)=\frac{\exp \left(\alpha_{j y} \zeta_{i}+\beta_{j y}\right)}{\sum_{k=0}^{r-1} \exp \left(\alpha_{j k} \zeta_{i}+\beta_{j k}\right)}
$$

for $y=0,1, \ldots, r-1$. Unlike the other models considered here, the nominal response model does not assume a particular ordering of the response categories. See Bock (1972, 1997) concerning the interpretation of the item response categories in terms of their relationship with the item response functions. The fmodelnrm in the ltbayes package evaluates the posterior of the latent trait of a nominal response model.

\section{References}

Andersen, E.B. (1970). Asymptotic properties of conditional likelihood estimates. Journal of the Royal Statistical Society: Series B, 32, 283-301.

Andersen, E.B. (1977). Sufficient statistics and latent trait models. Psychometrika, 42, 69-81. 
Andrich, D. (1978a). A rating formulation for ordered response categories. Psychometrika, 43, 561-573.

Andrich, D. (1978b). Application of a psychometric rating scale model to ordered categories which are scored with successive integers. Applied Psychological Measurement, 2, 581-594.

Andrich, D., \& Luo, G. (1993). A hyperbolic cosine latent trait model for unfolding dichotomous single-stimulus responses. Applied Psychological Measurement, 17, 253-276.

Baker, F.B., \& Kim, S.H. (2004). Item response theory: Parameter estimation techniques, 2nd edn. New York: Marcel-Dekker.

Barton, M.A., \& Lord, R.M. (1981). An upper asymptote for the threeparameter logistic item-response model. New Jersey: Educational Testing Service.

Birnbaum, A. (1968). Some latent trait models and their use in inferring an examinee's ability. In F. M. Lord \& M. R. Novick (Eds.) Statistical theories of mental test scores (pp. 397-479). Reading: Addison-Wesley.

Bock, R.D. (1972). Estimating item parameters and latent ability when responses are scored in two or more nominal categories. Psychometrika, 37, 29-51.

Bock, R.D. (1997). The nominal categories model. In W. J. van der Linden \& R. K. Hambleton (Eds.) Handbook of modern item response theory (pp. 33-49). New York: Springer.

Bock, R.D., \& Aitkin, M. (1981). Marginal maximum likelihood estimation of item parameters: Application of an EM algorithm. Psychometrika, 46, 443-459.

Bock, R.D., \& Lieberman, M. (1970). Fitting a response model from dichotomously scored items. Psychometrika, 35, 179197.

Chang, H. (1996). The asymptotic posterior normality of the latent trait for polytomous IRT models. Psychometrika, 61, 445463.

Chang, H., \& Stout, W. (1993). The asymptotic posterior normality of the latent trait in an IRT model. Psychometrika, 58, 37-52.

Chivers, C. (2012). MHadaptive: General Markov chain Monte Carlo for Bayesian inference using adaptive Metropolis-Hastings sampling. R package version 1.1-8. http://CRAN.R-project.org/ package $=$ MHadaptive

Curtis, S.M. (2012). mcmcplots: Create plots from MCMC output. R package version 0.4.1. http://CRAN.R-project.org/ package $=$ mcmcplots

Donovan, M.A., Drasgow, F., Probst, T.M. (2000). Does computerizing paper-and-pencil job attitude scales make a difference? New IRT analyses offer insight. Journal of Applied Psychology, 85, 305313.

Drasgow, F., \& Hulin, C.L. (1990). Item response theory. In M. D. Dunnette \& L. M. Hough (Eds.) Handbook of industrial and organizational psychology (Vol. 1, pp. 577-636). Palo Alto: Consulting Psychologists Press, Inc.

Fernández, X. (2013). ggmcmc: Graphical tools for analyzing Markov chain Monte Carlo simulations using Bayesian inference. R package version 0.5.1. http://CRAN.R-project.org/package $=$ ggmcmc

Geyer, C.J., \& Johnson, L.T. (2013). mcmc: Markov chain Monte Carlo. $\mathrm{R}$ package version 0.9-2. http://CRAN.R-project.org/ package $=$ mcmc

Grayson, D.A. (1988). Two-group classification in latent trait theory: Scores with monotone likelihood ratio. Psychometrika, 53, 383392.

Guion, R.M. (2011). Assessment, measurement, and prediction for personnel decisions. Taylor \& Francis.

Hambleton, R.K., Swaminathan, H., Rogers, J.H. (1991). Fundamentals of item response theory. Newbury Park: Sage.

Hemker, B.T., Sijtsma, K., Molenaar, I.W., Junker, B.W. (1996). Polytomous IRT models and monotone likelihood ratio of the total score. Psychometrika, 61, 679-693.
Hemker, B.T., Sijtsma, K., Molenaar, I.W., Junker, B.W. (1997). Stochastic ordering using the latent trait and the sum score in polytomous IRT models. Psychometrika, 62, 331-347.

Hemker, B.T., Van der Ark, L.A., Sijtsma, K. (2001). On measurement properties of continuation ratio models. Psychometrika, 66, 487506.

Highhouse, S. (2008). Stubborn reliance on intuition and subjectivity in employee selection. Industrial and Organizational Psychology, $1,333-342$.

Huynh, H. (1994). A new proof for monotone likelihood ratio for the sum of independent Bernoulli random variables. Psychometrika, $59,77-79$.

Johnson, T.R. (2013). Item response modeling with sum scores. Applied Psychological Measurement, 37, 638-652.

Johnson, T.R. (2014). Itbayes: Simulation-based Bayesian inference for latent traits of item response models. $\mathrm{R}$ package version 0.3 . http://CRAN.R-project.org/package=ltbayes

Lawshe, C.H., \& Bolda, R.A. (1958). Expectancy charts: I. Their use and empirical development. Personnel Psychology, 11, 353-365.

Lord, F.M. (1952). A theory of test scores (Psychometric Monograph No. 7). Richmond: Psychometric Corporation.

Lord, F.M. (1953). The relation of test score to the trait underlying the test. Educational and Psychological Measurement, 13, 517-548.

Lord, F.M. (1980). Applications of item response theory to practical testing problems. Hillsdale: Lawrence Erlbaum Associates.

Lord, F.M. (1983). Unbiased estimators of ability parameters, of their variance, and of their parallel-forms reliability. Psychometrika, 48 , 233-245.

Mair, P., \& Hatzinger, R. (2007). Extended Rasch modeling: The eRm package for the application of IRT models in R. Journal of Statistical Software, 20, 1-20.

Mair, P., Hatzinger, R., Maier, M.J. (2014). eRm: Extended Rasch Modeling. R package version 0.15-4. http://erm.r-forge.r-project. org/

Martin, A.D., Quinn, K.M., Park, J.-H. (2011). MCMCpack: Markov chain Monte Carlo in R. Journal of Statistical Software, 42, 1-21.

Masters, G.N. (1982). A Rasch model for partial credit scoring. Psychometrika, 47, 149-174.

Metropolis, N., Rosenbluth, A.W., Rosenbluth, M.N., Teller, A.H., Teller, E. (1953). Equations of state calculations by fast computing machine. Journal of Chemical Physics, 21, 1087-1091.

Muraki, E. (1990). Fitting a polytomous item response model to Likert-type data. Applied Psychological Measurement, 14, 59-71.

Muraki, E. (1992). A generalized partial credit model: Application of an EM algorithm. Applied Psychological Measurement, 16, 159176.

Patz, R.J., \& Junker, B.W. (1999a). A straightforward approach to Markov chain Monte Carlo methods for item response models. Journal of Educational and Behavioral Statistics, 24, 146-178.

Patz, R.J., \& Junker, B.W. (1999b). Applications and extensions of MCMC in IRT: Multiple item types, missing data, and rates responses. Journal of Educational and Behavioral Statistics, 21, 342-366.

Plummer, M., Best, N., Cowles, K., Vines, K. (2006). CODA: Convergence diagnosis and output analysis for MCMC. R News, 6, 711.

Raiche, G. (2014). irtProb: Utilities and probability distributions related to multidimensional person item response models. $\mathrm{R}$ package version 1.2. http://CRAN.R-project.org/package=irtProb

R Core Team. (2013). R: A language and environment for statistical computing. $R$ Foundation for Statistical Computing, Vienna, Austria. http://www.R-project.org/

Rizopoulos, D. (2006). 1tm: An R package for latent variable modeling and item response theory analysis. Journal of Statistical Software, $17,1-25$. 
Samejima, F. (1969). Estimation of ability using a response pattern of graded scores. Psychometrikka Monograph, 17.

Samejima, F. (1972). A general model for free-response data. Psychometrika Monograph, 18.

Schneider, R.J., Goff, M., Anderson, S., Borman, W.C. (2003). Computerized adaptive rating scales for measuring managerial performance. International Journal of Selection and Assessment, 11, 237-246.

Sijtsma, K., \& Hemker, B.T. (2000). A taxonomy of IRT models for ordering persons and items using simple sum scores. Journal of Educational and Behavioral Statistics, 25, 391-415.

Tutz, G. (1990). Sequential item response models with an ordered response. British Journal of Mathematical and Statistical Psychology, 43, 39-55.

Tutz, G. (1997). Sequential models for ordered responses. In W. J. van der Linden \& R. K. Hambleton (Eds.) Handbook of modern item response theory (pp. 139-152). New York: Springer-Verlag.

Van der Ark, L.A. (2001). Relationships and properties of polytomous item response theory models. Applied Psychological Measurement, 25, 273-282.

Van der Ark, L.A. (2005). Stochastic ordering of the latent trait by the sum score under various polytomous IRT models. Psychometrika, $70,283-304$.
Van der Ark, L.A., \& Bergsma, W.P. (2010). A note on stochastic ordering of the latent trait using the sum of polytomous item scores. Psychometrika, 75, 272-279.

Van der Linden, W.J., \& Pashley, P.J. (2010). Item selection and ability estimation in adaptive testing. In W. J. van der Linden \& C. A. W. Glas (Eds.) Elements of adaptive testing. Springer.

Verhelst, N.D., Glas, C.A.W., de Vries, H.H. (1997). A steps model to analyze partial credit. In W. J. van der Linden \& R. K. Hambleton (Eds.) Handbook of modern item response theory (pp. 123 138). New York: Springer-Verlag.

Verkulien, J. (2006). The Fisher information function for ideal point item response models for pick any/n data. Paper presented at the International Meeting of the Psychometric Society, Montréal, Canada.

Warm, T.A. (1989). Weighted likelihood estimation of ability in item response theory. Psychometrika, 54, 427-450.

Zickar, M.J., Gibby, R.E., Robie, C. (2004). Uncovering faking samples in applicant, incumbent, and experimental data sets: An application of mixed-model item response theory. Organizational Research Methods, 7, 168-90.

Zyphur, M.J., \& Oswald, F.L. (2013). Bayesian probability and statistics in management research: A new horizon. Journal of Management, 39, 5-13. 